See discussions, stats, and author profiles for this publication at: https://www.researchgate.net/publication/256596197

\title{
Physics Essays 1995
}

Data $\cdot$ September 2013

CITATIONS

0

1 author:

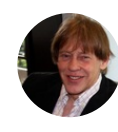

Wim Vegt

Technische Universiteit Eindhoven

6 PUBLICATIONS 10 CITATIONS

SEE PROFILE
READS

29

Some of the authors of this publication are also working on these related projects: 


\title{
A Continuous Model of Matter Based on AEONs
}

J.W. Vegt

\begin{abstract}
Within the domain of an electromagnetic model of matter it is supposed in this article that quantum mechanical probability waves are the observed relativistic effects of (auto-) confined electromagnetic waves. A relativistic coupling in the energy domain of the continuity equation results in the Schrodinger wave equation, while an extended coupling including the momentum domain (observed as "spin") results in the Dirac equation. It is demonstrated in Sec. 3 that the (relativistic) Dirac equation presumably originates from the Maxwell equations. Within the scope of this electromagnetic model of matter, "light," or more generally, "electromagnetic radiation," is regarded to be the building material of matter, which is effectively an inversion of the idea that light consists of elementary particles (photons). This postulated concept of inversion is extended to particles with a finite rest mass. The stability conditions for electromagnetic self-confinements (EONs) are obtained by the gravitational or electromagnetostatic forces or a combination of them, controlling the confinement.
\end{abstract}

Key words: auto-confined electromagnetic entities, electromagnetic waves, probability waves, relativity, Schrödinger equation, Dirac equation, phase-locked cavities

\section{INTRODUCTION: ELECTROMAGNETIC ENTITIES}

The acronym EON (electromagnetic entity) was first introduced by Wheeler ${ }^{(\mathbf{l})}$ in 1955 to introduce the concept of GEONs (gravitational electromagnetic entities), which describe the autoconfinements of electromagnetic radiation by self-generated gravity. AEONs (auto-confined electromagnetic entities) describe in general the concept of auto-confinements of electromagnetic radiation. AEONs include self-confined electromagnetic entities by gravity (GEONs) or by electromagnetostatic forces (EEONs, electromagnetostatic-confined electromagnetic entities).

The most important developments in particle physics are based on a fundamental discontinuity in the composition of matter that is implied by each underlying particle model. This article describes an electromagnetically continuous model of matter that does not use the concept of elementary particles, but of gravitationally or electromagnetostatically self-confined electromagnetic radiation in a perfect vacuum. The model should be regarded as a component of a concept for what may prove to be an alternative approach in elementary particle physics. An attempt is made to accommodate on physical grounds an intuitive awareness that the physical world is essentially a continuum. For the purposes of this model, light, rather, electromagnetic radiation in the more general sense of the term, is regarded as the building material of matter and of leptons, in particular.

\section{AN ELECTROMAGNETIC (RELATIVISTIC)} APPROACH TO THE SCHRÖDINGER EQUATION

The model assumes electromagnetic radiation in a perfect vacuum. Rather than working with the electric field intensity $\mathbf{E}$ and the magnetic induction B directly, it is usually more convenient to work in terms of the potentials. The scalar potential $\varphi$ and the vector potential $A$ are defined by

$$
\begin{gathered}
\mathbf{E}=-\operatorname{grad} \varphi-\partial \mathbf{A} / \partial t, \\
\mathbf{B}=\boldsymbol{\nabla} \times \mathbf{A} .
\end{gathered}
$$

If the 4-potential, related to the Lienard-Wiechert potentials, ${ }^{(2)}$ is defined by

$$
\varphi_{a}=\left[\frac{i \varphi}{c}, \mathbf{A}\right),
$$

then the electromagnetic field tensor or the Maxwell tensor is defined by

$$
F_{a b}=\partial_{b} \varphi_{a}-\partial_{a} \varphi_{b}
$$

in which $a, b$ assume the values $0,1,2,3$, respectively, where $i c t$ is the 0 component. Introducing the current density or source 4vector $j_{a}$ by

$$
j_{a}=(i c \rho, \mathbf{j})
$$

the Maxwell equations in relativistic units [c $=1$ and $G=1$ is 
used in Eqs. (6), (7), and (8)] can be written in the form

$$
\begin{gathered}
\partial_{b} F_{a b}=j_{a}, \\
\partial_{a} F_{b c}+\partial_{c} F_{a b}+\partial_{b} F_{c a}=0 .
\end{gathered}
$$

The Maxwell energy-momentum tensor $T_{a b}$ is

$$
T_{a b}=\frac{1}{4 \pi}\left[g^{c d} F_{a c} F_{a b}+\frac{1}{4} g_{a b} F_{c d} F^{c d}\right]
$$

in source-free regions. In Euclidian space the metric tensor equals

$$
g_{a b}=\delta_{a b},
$$

which means in general relativity the absence of a space-time curvature caused by mass. The equivalence of mass and energy in special relativity assumes that all forms of energy will act as sources for the gravitational field, which is expressed by (10). The Einstein tensor $G_{a b}$ is supposed to be proportional to the Maxwell energy-momentum tensor

$$
G_{a b}=\kappa T_{a b}
$$

where $x$ is a constant of proportionality, called the coupling constant and is equal to

$$
\kappa=\frac{8 \pi G}{c^{2}}
$$

where $G$ is the gravitational constant. Substituting (8) into (10) results in the Einstein-Maxwell equations

$$
G_{a b}=\frac{2 G}{c^{2}}\left[g^{\alpha d} F_{a c} F_{a b}+\frac{1}{4} g_{a b} F_{c d} F^{\alpha d}\right]
$$

To this point the theory is classical and well known. An introduction of a new concept in electromagnetism is done by introducing the complex vector wave function denoted by $\Phi(x, t)$ and the conjugated complex vector wave function $\Phi^{\prime \prime}(x, t)$, where

$$
\begin{aligned}
& \Phi(\mathbf{x}, t)=\left[\frac{\epsilon}{2}\right]^{1 / 2} \\
& \left(\nabla \times \mathbf{A}(\mathbf{x}, t)-\frac{i}{c}\left[\nabla \varphi(\mathbf{x}, t)+\frac{\partial \mathbf{A}(\mathbf{x}, t)}{\partial t}\right]\right) .
\end{aligned}
$$

In this equation, $\epsilon$ is the permittivity constant, and $c$ is the speed of light, which equals $(\epsilon \mu)^{-1 / 2}$. The complex vector functions are chosen such that the scalar product of both vector functions is equal to the relativistic electromagnetic mass density distribution $\rho_{\mathrm{PM}}(\mathbf{x}, t)$ (which equals $c^{-2}$ times the electromagnetic energy density) in the electromagnetic wave:

$$
\rho_{\mathrm{EM}}(\mathbf{x}, t)=\Phi(\mathbf{x}, t) \cdot \Phi^{*}(\mathbf{x}, t)
$$

The transport of electromagnetic energy is determined by the Poynting vector $\mathbf{S}(\mathbf{x}, t)$, which equals the cross product of both vector functions multiplied by $i c^{3}$ :

$$
\mathbf{S}(\mathbf{x}, t)=i c^{3}\left(\Phi^{*}(\mathbf{x}, t)\right) \times(\Phi(\mathbf{x}, t)) .
$$

In the absence of gravity Eqs. (14) and (15) equal Eqs. (A3) and (A4) in the Appendix. If the model is to have plausible foundations, electromagnetic radiation must possess material properties. Free electromagnetic radiation in no way satisfies this condition. While obeying Maxwell's laws, it does not satisfy the Schrödinger wave equation or the laws of inertia. (Self-) Confined electromagnetic radiation does, however, possess material properties.

Confined electromagnetic radiation exhibits the property of inertia, ${ }^{(3-5)}$ which describes the measured electromagnetic mass of longitudinal photons. It further satisfies, in first-order approximation, the law of inertia (Ref. 6, p. 172) as formulated by Newton, subject to the condition that the dimension of the self-confined radiation is much smaller than $c^{2} / a$, where $a$, the modulus of acceleration of the wave packet, which is demonstrated in (78) and Eqs. (A42) and (A50) in the Appendix, is restricted to the absence of gravity. In order to simplify the calculation of the relativistic effects, the starting point chosen for this model is a simplified model of external confinement consisting of perfectly reflecting mirrors (with adjustable curvature and negligible mass) within which a monochromatic beam of light is trapped, describing a phase-locked cavity. ${ }^{(4,5,7)}$ The mass of this confined electromagnetic wave is not negligible in this model. The monochromatic nature of the confined wave is essential to the entire model.

In a way comparable to free electromagnetic radiation, confined radiation satisfies the Lorentz transformation, which describes the relativistic effects that arise if an observer moves at a relative velocity $v$ with respect to the source of an electromagnetic wave, which is presented by

$$
F_{a b}^{\prime}=L_{c}^{a} F_{c d} L_{d}^{b}
$$

where $a, b, c, d$ assume the values 0 to 3 , respectively. $L_{a}^{b}$ is the Lorentz transformation matrix, given, for example, in (A57) for a transformation due to a relative velocity along the $x$-axis between observer and field configuration, and $F_{o d}$ is the fielddescribing matrix presented in (A27). In classical quantum mechanics, which is mainly wave mechanics, it is sometimes 
preferable to consider confined electromagnetic radiation as the superposition of Fourier components propagating in the opposite direction. This is illustrated in Eq. (A19). It will be clear that superposition of the Lorentz transformations of the Fourier components, propagating in opposite direction, has to be equal to the Lorentz transformation (A56), which is the usual presentation. This equality is demonstrated, for example, by the Lorentz transformations (A59), (A61), and (41) in which (41) is the result of the Lorentz transformation of the Fourier components, traveling in opposite directions.

In the most reduced example of plane monochromatic radiation, the confined radiation can be described by two plane waves traveling in opposite directions, confined by two perfect reflecting mirrors. When the movement is parallel to the confined beam, the observer simultaneously discerns an increased frequency of the beam propagating towards the observer and a decreased frequency of the beam propagating in the opposite direction. ${ }^{(8-10)}$ The transformation of the beam propagating in the same direction of the observer is indicated as ${ }^{-} L_{a}^{b}$, while the transformation of the beam moving in the opposite direction is indicated by ${ }^{+} L_{a}^{b}$. The averaged observed frequencies and energies of both beams, propagating in opposite directions, are increased according to (A10) and (A11) in the Appendix by a factor $+1 / 2 v^{2} / c^{2}$ in first-order approximation and are proportional to the observed kinetic energy $1 / 2 m_{\mathrm{E}} v^{2}$ (where $m_{\mathrm{E}}=W / c^{2}$ ) of the electromagnetic mass, which is accordingly classical mechanics. This is a characteristic of all kinds of confined radiation. $^{\text {(n) }}$

The Lorentz transformation of confined radiation is described by ${ }^{s} L_{a}^{b}$ in which $s$ differs in sign due to the described part of the confined wave. The corresponding tensor ${ }^{s} F_{c d}$ consists of a part ${ }^{-} F_{o d}$ describing the waves propagating in the same direction as the observer and a part ${ }^{+} F_{c d}$ describing the waves propagating in the opposite direction. The corresponding tensor $F_{c t}$ transforms as follows:

$$
F_{a b}^{\prime}={ }^{+} L_{c}^{a}{ }^{+} F_{c d}+L_{d}^{b}+{ }^{-} L_{c}^{a}-F_{c d}-L_{d}^{b} .
$$

The Lorentz transformation ${ }^{(11)}(17)$ is identical to (16) but offers some advantages.

A very simplified example of this concept is demonstrated in the Appendix in Eqs. (A9), (A16), and (A19). Making use of this basic principle, confined monochromatic radiation presents inertia (78) and obeys (20), Planck's law, and in a mode of selfconfinement the Schrodinger wave Eq. (56). Derivation of Planck's law for confined radiation (Ref. 7, p. 1531) is made under the assumption that the Lorentz transformation is valid in (slow) or nonaccelerated movements. In that case one can consider a confined electromagnetic field, for example, monochromatic radiation with frequency $f_{0}$ and energy $W_{0}$ confined between two perfect reflecting mirrors, which is compressed by moving one mirror slowly to the other such that no kinetic energy of the confined electromagnetic mass is introduced.
Moving both mirrors towards each other with a constant velocity $v$ means that work has to be done to counterbalance the radiation pressure, given in (A48) and (A51), which equals $1 / 2 w$, where $w$ is the energy density of the confined radiation, while simultaneously a rise in frequency occurs due to the Doppler shift. The distance between both mirrors is $l$ and their surface $\Lambda$. The time $\Delta t$ equals $2 l / c$, which again the reflected light requires to reach the moving reflecting mirror. During the time $\Delta t$ work $\Delta W$ has been done equal to

$$
\Delta W=1 / 2 w \Lambda v \Delta t=\frac{w \Lambda v l}{c}=\frac{v W_{0}}{c} .
$$

During the same interval $\Delta t$, the incident wave on the moving mirror is reflected with an increased frequency due to the Doppler shift, given by (A10), and equals

$$
f^{\prime}=f_{0}+\Delta f=\gamma\left(1+\frac{v}{c}\right) f_{0} .
$$

At low compression velocity $\gamma$ is nearly 1 . Combining (18) and (19) results in

$$
\Delta W=\frac{W_{0}}{f_{0}} \Delta f
$$

The term $\Delta f$ is the frequency shift after one complete reflection between both mirrors. Continuing the compression, the frequency shift also continues by discrete steps $\Delta f$ after each reflection of the moving mirror. This compression method has been applied, for example, in coupled high-power pulse lasers in which light intensities of $100 \mathrm{GW}$ in the ultraviolet region can now be reached. There is no theoretical limit for a maximum frequency or energy density, only a practical one. Mirrors are not able to compress the confined radiation to frequencies comparable with frequencies corresponding to elementary particles and an energy density desired for self-confinement. Only in phenomena like black holes can the desired compression forces occur.

The constant $W_{0} / f_{0}$ is indicated as $h_{\mathrm{E}}$ and is comparable to Planck's constant. The ratio $W_{0} / f_{0}$ is independent of the velocity $v$ of the moving mirror, which implies that independent of the velocity of compression, the frequency of confined radiation increases proportionally to the energy of the system. For a monochromatic system of confinement of radiation with homogeneous energy density, linear integration of (20) results in (21). Introducing a system of asymptotic infinite cubes with asymptotic infinite small sizes, all confining radiation of different energy density leads to a more general law (21) for confined radiation with an arbitrary energy density, derived by integrating (20) over an arbitrary volume of (self-) confined radiation:

$$
W=h_{\mathrm{E}} f,
$$


where $W$ is the total (self-) confined electromagnetic energy, and $f$ is the frequency of the (self-) confined monochromatic wave. ${ }^{(12)}$ In a system of self-confinement the corresponding frequency, wavelength, and associated mass are determined by geometric conditions. ${ }^{(11,13)}$

In circumstances of self-confinement, electromagnetic radiation satisfies the Schrödinger wave Eq. (56) when energy densities are sufficiently high ${ }^{(1)}$ (of the order of $10^{73} \mathrm{~J} / \mathrm{m}^{3}$ ), as demonstrated in (117). For this purpose, the starting point is the model of external radiation confinement within a system of perfectly reflecting mirrors, after which the aspect of (gravitational or electromagnetostatic) self-confinement is introduced at a later stage. The mirror system has been chosen such that a vibration mode of electromagnetic radiation with frequency $\omega_{0}$ satisfies the consequent boundary conditions. In this example, if the system of mirrors is at rest with respect to an observer, an electric field vector is measured which is equal to

$$
\begin{aligned}
\mathbf{E}(\mathbf{x}, t) & =\frac{i c\left(\Phi^{*}-\Phi\right)}{(2 \epsilon)^{1 / 2}} \\
& =\frac{-\mathbf{E}_{0}}{4} \int\left(e^{i \omega t}-e^{-i \omega}\right)\left(e^{i k \cdot \mathbf{x}}-e^{-i k \cdot x}\right) \delta\left(\omega_{0}\right) d \omega
\end{aligned}
$$

and in which $E_{0}$ is the amplitude of the electrical field intensity. The magnetic field vector observed is equal to

$$
\begin{aligned}
\mathbf{H}(\mathbf{x}, t) & =\frac{\left(\Phi^{*}+\Phi\right)}{\mu(2 \varepsilon)^{1 / 2}} \\
& =\frac{\mathbf{e}_{s} \times \mathbf{E}_{0}}{(2 \mu / \varepsilon)^{1 / 2}} \int\left(e^{i \omega t}+e^{-i \omega}\right)\left(e^{i \mathbf{k} \cdot \mathbf{x}}+e^{-i k \cdot x}\right) \delta\left(\omega_{0}\right) d \omega,
\end{aligned}
$$

where $e_{s}$ is the unit vector in the direction of wave propagation and $\mu$ the permeability. The electric and magnetic field intensities (22) and (23) are measured by an observer at rest with respect to the system of mirrors. An observer, moving relative to the mirror's coordinate system, discerns a transformed electric and magnetic field intensity, frequency, and wavelength. Introducing the 4-wave vector

$$
k_{a}=\left(\frac{i \omega}{c}, \mathbf{k}\right)
$$

the transformed frequency and wavelength ${ }^{(14)}$ are presented by

$$
{ }^{\prime} k_{a}^{\prime}={ }^{+} L_{b}^{a}+k_{b}+{ }^{-} L_{b}^{a}-k_{b},
$$

which one-dimensional transformation is given in (A12) and (A13). We define the Lorentz contraction term $\gamma$,

$$
\gamma=\left(1-\frac{\mathbf{v}_{G} \cdot \nabla_{G}}{c^{2}}\right)^{-1 / 2},
$$

where $\mathbf{v}_{G}$ is the velocity of the observer relative to the coordinate system of the confining mirrors. Using (17), (22), (25), and (26), the transformed electric field intensity ${ }^{(14)}$ is presented by

$$
\mathbf{E}^{\prime}\left(\mathbf{x}^{\prime}, t^{\prime}\right)=\gamma\left[\mathbf{E}_{0} \sin \beta \sin \alpha+\frac{\nabla_{G} \times \mathbf{e}_{s} \times \mathbf{E}_{0}}{c} \cos \beta \cos \alpha\right] .
$$

The phase $\beta$ is given by

$$
\beta=\gamma\left[\omega_{0} t^{\prime}+\frac{\left(\nabla_{G} \cdot \mathbf{e}_{s}\right)\left(\mathbf{k} \cdot \mathbf{x}^{\prime}\right)}{c}\right],
$$

where $\omega_{0}$ is the nontransformed frequency, mostly indicated as rest frequency. Phase $\alpha$ is given by

$$
\alpha=\gamma\left(\mathbf{k} \cdot \mathbf{x}^{\prime}+\frac{\left(\mathbf{v}_{G} \cdot \mathbf{e}_{S}\right) \omega_{0} t^{\prime}}{c}\right] .
$$

This result is also demonstrated in a one-dimensional example in the Appendix in Eqs. (A19) and (A20). Phases $\beta$ and $\alpha$ in (28) and (29) are Lorentz-invariant parameters, which follows from the reverse transformation of the observer's coordinates $\left(\Sigma^{\prime}\right)$ to

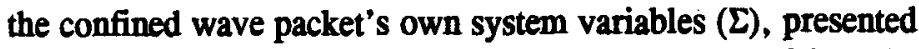
in the one-dimensional example in (A25) and (A26). The observer in $\left(\Sigma^{\prime}\right)$ measures an electromagnetic wave with an apparent phase velocity (Ref. 3, p. 145) $\mathbf{\nabla}_{\beta}$, which is determined by (28):

$$
\nabla_{B} \cdot \nabla_{G}=c^{2},
$$

which equation is comparable to (A22) in the Appendix. From (30) it follows that the phase velocity of a confined electromagnetic wave packet traveling at a velocity $\nabla_{G}$ with respect to an observer is always measured by the latter as greater than the velocity of light as a result of the relativistic transformations (17) and (25). This is accordingly the phase velocity of quantum mechanical probability waves describing elementary particles. Because the phase velocity is not related to the velocity of transport of information or energy and mass, (30) does not contradict the principle of relativity. Phase $\beta$ in (28) represents a relativistically transformed wavelength $\lambda$ (in the direction of propagation of the wave packet), which is measured by a stationary observer in the coordinate system $\left(\Sigma^{\prime}\right)$ with respect to which a confined electromagnetic monochromatic wave packet moves at a velocity $\nabla_{G}$, yielding 


$$
\lambda=\frac{2 \pi c}{\gamma \mathbf{k} \cdot \nabla_{G}} .
$$

Combining the Einstein relation $W_{0}=m_{0} c^{2}$, where $W_{0}$ is the rest energy and $m_{0}$ is the rest mass, with (21) and the fact that the modulus of the wave vector $k$ is equal to $\omega / c$, there follows from (31) a relation ${ }^{(15)}$ for the observed relativistic 4-wavelength $\lambda_{a}:$

$$
\lambda_{a}=h_{E} / p_{a}
$$

which equation is equal to (A24) for $a=1$. In (32) $\lambda_{a}$ is the observed wavelength in coordinate direction $a$, the zero component $\lambda_{0}=-i c T$ is the time component of the wavelength, and $h_{\mathrm{E}}$ is the constant defined in (21). The term $p_{a}$ is the relativistic momentum 4-vector of the confined electromagnetic monochromatic wave and is equal to the product of the relativistic mass $\gamma W_{d} / c^{2}$ and the 4-vector velocity $\left(i c, v_{G}\right)$. Relation (32) represents the observed relativistic effect of a confined electromagnetic monochromatic wave derived from (17) and (25) and shows a characteristic correspondence to quantum mechanical probability waves, describing elementary particles.

The force, operating on confined electromagnetic radiation, can be derived from the tension tensor $\overline{\overline{\mathbf{t}}}$, which is a subtensor in (A49), and the force Eq. (A48). The momentum 4-vector is defined by

$$
p^{a}=\left(\frac{i W}{c}, \mathbf{p}\right)
$$

The transformed potential 4-vector is presented by

$$
{ }^{s} \varphi_{a}^{\prime}={ }^{+} L_{b}^{a}+\varphi_{b}+-L_{b}^{a}-\varphi_{b} .
$$

Using (3), (13), and (34), the transformed vector wave function $\Phi^{\prime}\left(\mathbf{x}^{\prime}, t^{\prime}\right)$ is presented by

$$
\begin{aligned}
& \Phi^{\prime}\left(\mathbf{x}^{\prime}, t^{\prime}\right)=(\epsilon / 2)^{1 / 2} \\
& {\left[\nabla \times \mathbf{A}^{\prime}\left(\mathbf{x}^{\prime}, t^{\prime}\right)-\frac{i}{c}\left(\nabla \varphi^{\prime}\left(\mathbf{x}^{\prime}, t^{\prime}\right)+\frac{\partial \mathbf{A}^{\prime}\left(\mathbf{x}^{\prime}, t^{\prime}\right)}{\partial t^{\prime}}\right]\right) .}
\end{aligned}
$$

Making use of (14) and (15), the pseudo-Poynting 4-vector, which equals $i c T^{a 0}$ [presented in the Appendix in (A51) and related to the example in Fig. 1] is defined by

$$
\begin{aligned}
S^{a} & =\left(i c\left(w_{D}+w_{S}\right), \mathbf{S}\right) \\
& =i c^{3}\left[\left(\Phi^{*}(\mathbf{x}, t) \cdot \Phi(\mathbf{x}, t)\right),\left(\Phi(\mathbf{x}, t) \times \Phi^{*}(\mathbf{x}, t)\right)\right]
\end{aligned}
$$

The energy density $W_{\mathrm{D}}$ describes the dynamic radiation part of the confined electromagnetic phenomena, excluding the mostly static energy part $w_{\mathrm{s}}$ confining the system. The transformed Poynting 4-vector is presented by

$$
S^{a^{\prime}}=\left(i c\left(w_{\mathrm{D}}^{\prime}+w_{\mathrm{S}}^{\prime}\right), \mathbf{S}^{\prime}\right)=i c^{3}\left[\left(\Phi^{*^{\prime}} \cdot \Phi^{\prime}\right),\left(\Phi^{\prime} \times \Phi^{*^{\prime}}\right)\right]
$$

Only under the condition that the energy density is comprised of the dynamic and the confining static parts does the Poynting pseudo 4-vector in (37) transform like a real 4-vector with Lorentz-invariant modulus. Substitution of (35) in (37), and using (34), results in an alternative notation for the transformation of the Poynting 4-vector:

$$
{ }^{s} S_{a}^{\prime}={ }^{+} L_{b}^{a}+S_{b}+{ }^{-} L_{b}^{a}-S_{b}
$$

The transformation (38) is for confined radiation in perfect balance identical to the transformation (A56) for the index $d=$ 0 in the Appendix. The momentum 4-vector of the confined radiation can be determined with the Poynting 4-vector (36) by (A50), which equals

$$
p^{a}=\frac{i}{c} \int_{\text {Vol }} T^{a} d V=\frac{1}{c^{2}} \int_{\text {vol }} S^{a} d V
$$

The momentum 4-vector for confined electromagnetic radiation is related by (39) to the Poynting 4-vector of the electromagnetic system. The introduction of a Poynting pseudo 4-vector for confined electromagnetic phenomena and its relation to the momentum 4-vector, given by (39), is an aspect in classical electromagnetism that relates in an important way classical mechanics to quantum mechanics. This relation is an elementary contribution to the particlelike property of confined radiation, which implies that confined monochromatic radiation obeys the Schrödinger wave equation. It is important to note that only because the Poynting pseudo 4-vector in (38) obeys the Lorentz transformation can the continuity equation be written as a Schrödinger equation for confined electromagnetic radiation. Making use of (38) and (39), the transformation of the momentum 4-vector for confined radiation is represented by

$$
p_{a}^{\prime}=L_{b}^{a} p_{b}
$$

Equation (40) offers a significant result. Electromagnetic confinements in equilibrium or electromagnetic self-confinements appear to transform identically to elementary particles.

Formulas (37) and (38) are determined by calculating the Poynting 4-vector of perfect confined electromagnetic radiation. In the case of Lorentz-invariant transformations of the Poynting 4-vector modulus, the parameter of a system in equilibrium must be transformed. ${ }^{(8-10)}$ In the model of external radiation confine- 
ment by a system of mirrors (Fig. 1), this is achieved, for example, by applying an opposite electric charge on a pair of mirrors, which compensates for the radiation pressure between the two massless mirrors facing each other.

If the distance between the two mirrors decreases slightly, the radiation pressure as well as the frequency increase [Eqs. (18) and (19)], and the repulsive radiation pressure overcomes the attractive electrostatic force and vice versa. In this way the dynamic system is stable.

The Poynting vector and the energy density of the dynamic field with frequency $\omega$ are denoted by $S_{\mathrm{D}}$ and $w_{\mathrm{D}}$, respectively, and for the static field, which compensates for the repulsive radiation pressure on the two mirrors, these two parameters are denoted by $S_{\mathrm{s}}$ and $w_{\mathrm{s}}$, respectively. It is important to realize that the confined dynamic field ${ }^{(11,14)}$ with frequency $\omega$ transforms differently from the (static) field describing the confining system itself due to the different orientation of the fields relative to the moving observer.

A dynamic Poynting pseudo 4-vector, describing the frequency-dependent part of the pseudo-Poynting 4-vector, can be assigned to a monochromatic electromagnetic wave packet, externally confined in a system of mirrors and represented in its simplest form in (22) and (23). The transformation of the Poynting pseudo 4-vector can be performed more easily by using a notation reported earlier by Pauli, ${ }^{(16)}$ which split up the confined monochromatic wave in perfect symmetric parts related to the ${ }^{+} L$ and $-L$ transformations. Using the Pauli notation and (38), the transformed Poynting 4-vector is represented by

$$
\begin{aligned}
& \left(\mathbf{S}_{D}^{\prime}+\mathbf{S}_{S}^{\prime}\right)+i\left(w_{D}^{\prime}+w_{S}^{\prime}\right) c=\gamma^{2}\left[\left[1+\frac{\nabla_{G} \cdot \nabla_{G}}{c^{2}}\right] S_{D}+\frac{S_{S}}{\gamma^{2}}\right. \\
& \left.-2 \nabla_{G} w_{D}+i c\left[\left(1+\frac{\nabla_{G} \cdot \nabla_{G}}{c^{2}}\right] w_{D}+\frac{w_{S}}{\gamma^{2}}-\frac{2 \nabla_{G} \cdot S_{D}}{c^{2}}\right]\right] .
\end{aligned}
$$

This result is identical to the result obtained in the classical way from the transformation of the energy-momentum tensor (A56), demonstrated in (A59) and (A61) in the Appendix. The relativistic notation (41) for the Poynting 4-vector of a monochromatic electromagnetic wave packet leads to a relativistic equation, which demonstrates a remarkable correspondence with the Schrödinger, Klein-Gordon, and Dirac equations. The derivation for the Klein-Gordon equation is essentially based on Eqs. (38) and (39), to which the wavelike nature is introduced later by applying (32). For this reason, the Klein-Gordon equation also gives solutions for negative energy. The Dirac Eq. (102) corresponds with the Schrodinger wave equation at nonrelativistic velocities. ${ }^{(1)}$ This is not in contradiction with the relativistic origin of (41) because it is a well-known aspect that relativity is demonstrated most significantly at nonrelativistic velocities. For example, the phenomenon of mass, which is a pure relativistic effect of energy, is demonstrated most significantly at rest on a scale or at nonrelativistic velocities by its inertia. At relativistic velocities momentum and energy, or its

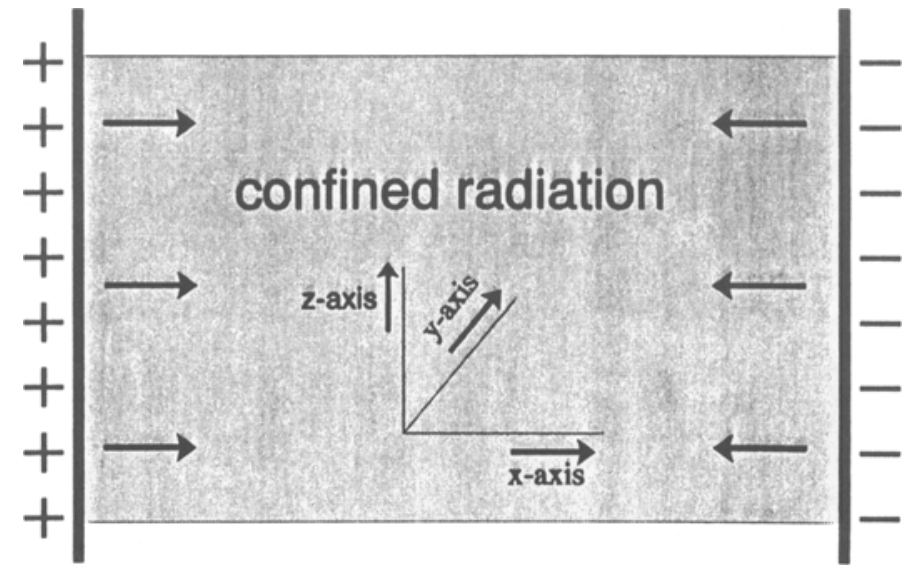

Figure 1. One-dimensional electromagnetic confinement in equilibrium.

equivalence mass, are not as easy to separate. In this article the results of (41) are considered, restricted to electromagnetic confinements at nonrelativistic velocities relative to the observer, and for that reason may only result in a Schrodinger-like wave equation.

The Schrödinger wave equation for (self-) confined electromagnetic radiation will be derived from the law of continuity below. The law of continuity for electromagnetic radiation, generally termed Poynting's theorem and given in (A63), which describes the law of conservation of electromagnetic energy, is presented in the observer's system of coordinates in vacuo by

$$
\nabla^{\prime} \cdot \mathbf{S}(\mathbf{x}, t)=-\frac{\partial w^{\prime}\left(\mathbf{x}^{\prime}, t^{\prime}\right)}{\partial t^{\prime}}
$$

In this equation $S^{\prime}\left(x^{\prime}, t\right)$ is Poynting's vector and $w^{\prime}\left(x^{\prime}, t\right)$ the electromagnetic energy density observed in the coordinate system of the observer. In the case of nonrelativistic velocities, substitution of (41) in (42) results in two equations in which the momentum and the energy, derived from the Poynting 4-vector, can be regarded as separate variables:

$$
\begin{gathered}
\frac{1}{2} \nabla^{\prime} \cdot\left(\mathbf{S}_{D}+\mathbf{S}_{S}\right)+\frac{v_{G}^{2}}{2 c^{2}} \nabla^{\prime} \cdot\left(\mathbf{S}_{D}-\mathbf{S}_{S}\right)=-\frac{1}{c^{2}} \frac{\partial\left(\nabla_{G} \cdot \mathbf{S}_{D}\right)}{\partial t^{\prime}} \\
\nabla^{\prime} \cdot\left(\nabla_{G} w_{D}\right)=-\frac{1}{2} \frac{\partial\left(w_{D}+w_{S}\right)}{\partial t^{\prime}}-\frac{\nabla_{G} \cdot \nabla_{G}}{2 c^{2}} \frac{\partial\left(w_{D}-w_{S}\right)}{\partial t^{\prime}} .
\end{gathered}
$$

In the case of nonrelativistic velocities, Eq. (43) can be regarded as a momentum density equation and (44) as an energy density equation. In the case of relativistic velocities, splitting 
(43) and (44) from (42) is only permitted under restricted conditions. At nonrelativistic velocities under certain conditions, Eq. (44) changes into the conventional Schrödinger equation for (self-)confined electromagnetic radiation. Bosons, described by symmetric wave functions, as well as fermions, described by antisymmetric wave functions, obey the Schrödinger wave equation. It is well known that electromagnetic phenomena can be described in terms of photons which belong to the boson group. A transition from bosons to fermions is not allowed in classical quantum mechanics. In a two-particle problem bosons are described by a symmetric wave function, in which the positions of particles 1 and 2 are represented by the vectors $\mathbf{r}_{1}$ and $\mathbf{r}_{2}$, respectively:

$$
\boldsymbol{\Psi}\left(\mathbf{r}_{1}, \mathbf{r}_{2}\right)=\Psi\left(\mathbf{r}_{2}, \mathbf{r}_{1}\right)
$$

Fermions are described by an antisymmetric wave function, which is represented in a two-particle problem as

$$
\Psi\left(r_{1}, r_{2}\right)=-\Psi\left(r_{2}, r_{1}\right)
$$

Because free electromagnetic waves are described by bosons, it would be a reasonable assumption that electro-magnetic entities (EONs) behave like bosons. However, this contradicts (44), which describes boson- as well as fermion-like behavior. By exchanging two particles, described by EONs, the relative velocities between the observer and the particles may change in sign, which is observed as a change in sign of the wave function, because the left of (44) only represents the relativistic part of the Poynting vector. The Lorentz matrices ${ }^{+} L$ and $-L$ exchange. By analogy with (13) the complex conjugated scalar functions $\Psi\left(x^{\prime}, t^{\prime}\right)$ and $\Psi^{*}\left(x^{\prime}, t^{\prime}\right)$ are introduced, yielding

$$
\tilde{\rho}_{\mathrm{EM}}\left(\mathbf{x}^{\prime}, t^{\prime}\right)=\Psi\left(\mathbf{x}^{\prime}, t^{\prime}\right) \Psi^{*}\left(\mathbf{x}^{\prime}, t^{\prime}\right)
$$

where $\tilde{\rho}_{\mathrm{EM}}\left(\mathbf{x}^{\prime}, t^{\prime}\right)$, just as in (14), is the averaged electromagnetic relativistic mass density. The function $\Psi\left(x^{\prime}, t^{\prime}\right)$, by definition, is complex and satisfies

$$
\Psi\left(\mathbf{x}^{\prime}, t^{\prime}\right)=\left[\frac{\epsilon}{2}\right]^{1 / 2}\left[\tilde{B}\left(\mathbf{x}^{\prime}, t^{\prime}\right)+\frac{i E\left(\mathbf{x}^{\prime}, t^{\prime}\right)}{c}\right]
$$

in which $\tilde{B}\left(\mathbf{x}^{\prime}, t^{\prime}\right)$ and $\tilde{E}\left(\mathbf{x}^{\prime}, t^{\prime}\right)$ are the effective values of the modulus of the magnetic induction and the electric field intensity, respectively, calculated across a microcube with the dimensions of half a wavelength. In the special case of the effective electric and magnetic field intensities calculated across a microcube, the position and time dependence of the function $\Psi\left(x^{\prime}, t^{\prime}\right)$ can be defined for a monochromatic electromagnetic wave packet with frequency $\omega_{0}$ with the aid of (22), (23), (27), (28), and (48) as follows:

$$
\Psi\left(\mathbf{x}^{\prime}, t^{\prime}\right)=\tilde{\Psi}_{\mathbf{R}}\left(\mathbf{x}^{\prime}, t^{\prime}\right) e^{i \beta}
$$

In (49) the function $\boldsymbol{\Psi}\left(\mathrm{x}^{\prime}, t^{\prime}\right)$ is represented by the product of a real function, denoted by $\tilde{\Psi}_{R}\left(x^{\prime}, t^{\prime}\right)$, which is equal to the root of the electromagnetic mass density averaged across a microcube with the dimension of the wavelength $\lambda$, and the term $e^{i \beta}$ which, if the confined electromagnetic wave is at rest with respect to the observer, is only determined by the fundamental frequency $\omega_{0}$ of the confined wave. Subject to the condition of nonrelativistic velocities and the assumption that the confined radiation energy density with frequency $\omega$ averaged across a microcube is equal to the static energy density of confinement, using the Einstein relation, averaged over a microcube, $\tilde{w}=\tilde{\rho}_{\mathrm{EM}} c^{2}$ and substitution of $(47)$ in (44) results in

$$
\Psi^{*} \nabla^{\prime} \cdot\left(\mathbf{v}_{G} \Psi\right)+\left(\Psi \mathbf{v}_{G}\right) \cdot \nabla^{\prime} \Psi^{*}=-\Psi \frac{\partial \Psi^{*}}{\partial t^{\prime}}-\Psi^{*} \frac{\partial \Psi}{\partial t^{\prime}}
$$

For a confined monochromatic electromagnetic wave with a rest frequency $\omega_{0}$, traveling at a velocity $v_{G}$ with respect to the observer and using (49) and (28) we have

$$
\nabla^{\prime} \Psi\left(\mathbf{x}^{\prime}, t^{\prime}\right)=e^{i \beta} \nabla^{\prime} \tilde{\Psi}_{R}\left(\mathbf{x}^{\prime}, t^{\prime}\right)+\frac{i \gamma \omega \mathbf{v}_{G}}{c^{2}} \Psi\left(\mathbf{x}^{\prime}, t^{\prime}\right)
$$

Substitution of (51) in (50) results in

$$
\frac{c^{2}}{i \gamma \omega} \nabla^{2^{\prime}} \Psi-\frac{c^{2} e^{i \beta}}{i \gamma \omega} \nabla^{2^{\prime}} \tilde{\Psi}_{R}-2 i \gamma \omega\left[1+\frac{v_{G}^{2}}{2 c^{2}}\right) \Psi=-2 \frac{\partial \Psi}{\partial t^{\prime}}
$$

Using Einstein's relation $W=m_{E} c^{2}=\hbar_{E} \omega$ with $\hbar=h_{E} / 2 \pi$, in which $h_{E}$ is introduced into Eq. (21) and $\omega_{0}$ is the rest frequency of the confined electromagnetic wave, (52) becomes

$$
-\frac{\hbar_{E}^{2}}{2 m_{E}} \nabla^{2} \Psi+\frac{\hbar_{E}^{2} e^{i \beta}}{2 m_{E}} \nabla^{2^{\prime}} \tilde{\Psi}_{R}-m_{E} c^{2}\left(1+\frac{v_{G}^{2}}{2 c^{2}}\right) \Psi=i \hbar_{E} \frac{\partial \Psi}{\partial t^{\prime}}
$$

Here, $m_{E}$ is the relativistic mass of the confined electromagnetic energy and is equal to $\gamma m_{0}$, where $m_{0}$ is the rest mass of the confined electromagnetic field energy. The term $m_{E} c^{2}$ is the internal energy $V_{0}$ of the confined electromagnetic radiation. The change of the external potential energy $V_{\mathrm{PE}}$ in an external force field is equal to the opposite change in the kinetic energy $1 / 2 m_{E} v_{G}^{2}$. Applying this in (53) results in 


$$
-\frac{\hbar_{E}^{2}}{2 m_{E}} \nabla^{2^{\prime}} \Psi+\frac{\hbar_{E}^{2} e^{i \beta}}{2 m_{E}} \nabla^{2^{\prime}} \tilde{\Psi}_{R}-V_{0} \Psi+V_{P E} \Psi=i \hbar_{E} \frac{\partial \Psi}{\partial t^{\prime}}
$$

To reduce (54) to a Schrodinger-type wave equation, a selfconfinement of the electromagnetic radiation is assumed in this model. This self-confinement can occur in several ways. A basic model assumes a self-confinement of electromagnetic radiation by gravitational waves generated by the electromagnetic energy itself. ${ }^{(1)}$

In the case of a gravitational confinement (or an electromagnetostatic confinement, which must fulfill the same conditions), it is assumed that, according to the basic principle of general relativity, any arbitrary energy $W$ also represents a mass $m$, which generates a gravitational field. In general relativity this effect is indicated by the coupling constant $\alpha$ in (11). Comparable to general relativity, a confined electromagnetic energy $W$ generates a gravitational field in a manner comparable to a material mass $m=W / c^{2}$. In the case of gravitational selfconfinement (or electromagnetostatic confinement), in this model the second term in (54) represents the internal potential energy $V_{\mathrm{Pl}}$, which occurs as a result of the interaction between gravitational confining forces and electromagnetic repulsive forces, given in (116). It follows from (116) that the second term in (54) vanishes at equilibrium. When the gravitational confining forces are in equilibrium with the repulsive electromagnetic radiation forces, the second term in (54) equals zero and (54) changes into

$$
-\frac{\hbar_{E}^{2}}{2 m_{E}} \nabla^{2^{\prime}} \Psi-V_{0} \Psi+V_{P E} \Psi=i \hbar_{E} \frac{\partial \Psi}{\partial t^{\prime}}
$$

In the event of interaction with the surroundings, the second term in (54) represents the internal potential energy $V_{F}$ and (54) changes to

$$
-\frac{\hbar_{E}^{2}}{2 m_{E}} \nabla^{2^{\prime}} \Psi+V_{\mathrm{P}} \Psi-V_{0} \Psi+V_{\mathrm{PE}} \Psi=i \hbar_{E} \frac{\partial \Psi}{\partial t^{\prime}}
$$

Equation (56) demonstrates that at nonrelativistic velocities, where the energy and the momentum are observed as separated quantities, this equation is a special notation for (A64) in the Appendix in which circumstance the Schrödinger equation controls the energy domain.

\section{THE DIRAC EQUATION RELATED TO THE MAXWELL EQUATIONS}

In what is undoubtedly one of the great papers in physics of this century, Dirac set up a relativistic wave equation that avoids the difficulties of negative probability density of the KleinGordon equation, and describes naturally the spin of the electron. Until Pauli and Weisskopf ${ }^{(18)}$ reinterpreted the KleinGordon equation, it was believed that this Dirac equation was the only valid relativistic equation. It is now recognized that the
Dirac equation and the Klein-Gordon equations are equally valid. The Dirac equation governs particles of spin-1/2, the Klein-Gordon equation those of spin-0 (Ref. 17, p. 349). Autoconfined electromagnetic entities (AEONs) presents a characteristic effect indicated as "spin." Spin is a fundamental key in relativistic quantum mechanics. ${ }^{(9,15,19-21)}$ Dirac with his relativistic equation for the electron was the first, as he put it, to wed quantum mechanics and relativity. The consequence of negative energies that the Dirac equation presents when it is solved introduces the existence of antiparticles, necessary to wed quantum mechanics and relativity. ${ }^{(22)}$ This can be worked out for any "spin" as shown by Pauli and Weisskopf. ${ }^{(18)}$ In this article, electromagnetic radiation is considered as the building material of a simultaneous particle-antiparticle combination, ${ }^{(9)}$ which can under special conditions split into two separate independent particles, both consisting of self-confined electromagnetic radiation. ${ }^{(8,19.23)}$ The Dirac equation ${ }^{(12,17)}$ can be derived from the continuity Eq. (42) or the Maxwell Eq. (60). The continuity equation equals

$$
\nabla \cdot S(x, t)=-\frac{\partial w(x, t)}{\partial t}
$$

Substituting (14) and (15) in (57) results in

$$
i c \nabla^{\prime} \cdot\left(\Phi^{*} \times \Phi\right)=-\frac{\partial\left(\Phi \cdot \Phi^{*}\right)}{\partial t}
$$

which can be written as

$$
i c \Phi \cdot\left(\nabla^{\prime} \times \Phi^{*}\right)-i c \Phi^{*} \cdot\left(\nabla^{\prime} \times \Phi\right)=-\Phi \cdot \frac{\partial \Phi^{*}}{\partial t}-\Phi^{*} \cdot \frac{\partial \Phi}{\partial t}
$$

Equation (59) can be split into two identical equations for $\Phi$ and $\Phi *$, respectively, which only are distinguished in sign:

$$
\nabla^{\prime} \times \Phi=-\frac{i}{c} \frac{\partial \Phi}{\partial t}
$$

Substituting Eq. (A3) in the Appendix in (60) demonstrates that the Maxwell Eqs. (A29) and (A32) follow straight from the continuity Eq. (57) (Ref. 18, p. 201). To transform the electromagnetic vector wave function $\Phi$ into a scalar (spinor or onedimensional matrix) representation, the Pauli spin matrices are introduced:

$$
\sigma_{\mathrm{X}}=\left[\begin{array}{ll}
0 & 1 \\
1 & 0
\end{array}\right], \quad \sigma_{\mathrm{Y}}=\left[\begin{array}{cc}
0 & -i \\
i & 0
\end{array}\right], \quad \sigma_{\mathrm{z}}=\left[\begin{array}{rr}
1 & 0 \\
0 & -1
\end{array}\right],
$$


which matrices are presented by the vector notation $\sigma$. The Pauli spin matrices obey the equation

$$
\sigma_{a} \sigma_{b}=i \epsilon_{a b c} \sigma_{c}+\delta_{a b}
$$

in which $\epsilon_{a b c}$ is the Levi-Civita tensor whose value is +1 or -1 if $a b c$ is an even or odd permutation, respectively. The electromagnetic vector wave function $\Phi$ is transformed into the matrix representation $\tilde{U}$ by the scalar product of the Pauli matrices $\sigma$ and the vector wave function $\Phi$ :

$$
\tilde{U}=\sigma \cdot \Phi=\left[\begin{array}{cc}
\Phi_{Z} & \Phi_{X}-i \Phi_{Y} \\
\Phi_{X}+i \Phi_{Y} & -\Phi_{Z}
\end{array}\right]=\left(\begin{array}{ll}
u_{3} & u_{1} \\
u_{4} & u_{2}
\end{array}\right) .
$$

For a more common notation in the continuity equation the spinor $\bar{U}$ is presented as

$$
U=\left(\begin{array}{l}
u_{1} \\
u_{2} \\
u_{3} \\
u_{4}
\end{array}\right)=\left(\begin{array}{c}
\Phi_{X}-i \Phi_{Y} \\
-\Phi_{Z} \\
\Phi_{Z} \\
\Phi_{X}+i \Phi_{Y}
\end{array}\right)
$$

Both sides of Eq. (60) are multiplied scalar by the Pauli spin matrices

$$
\sigma \cdot\left(\nabla^{\prime} \times \Phi\right)=-\frac{i \sigma}{c} \cdot\left(\frac{\partial \Phi}{\partial t}\right)
$$

From Eq. (62) it follows that

$$
(\sigma \cdot \nabla)(\sigma \cdot \Phi)=\delta_{a b}(\nabla \cdot \Phi)+i \sigma \cdot(\nabla \times \Phi)
$$

Substituting (66) in (65) results in

$$
(\sigma \cdot \nabla)(\sigma \cdot \Phi)-\delta_{a b}(\nabla \cdot \Phi)=\frac{\sigma}{c} \cdot\left[\frac{\partial \Phi}{\partial t}\right]
$$

From (61), (63), and (67) follows the equation

$$
(\sigma \cdot \nabla) \tilde{U}=\left(\begin{array}{cc}
\frac{\partial}{\partial z} & \left(\frac{\partial}{\partial x}-i \frac{\partial}{\partial y}\right) \\
\left(\frac{\partial}{\partial x}+i \frac{\partial}{\partial y}\right) & -\frac{\partial}{\partial z}
\end{array}\right]\left(\begin{array}{ll}
u_{3} & u_{1} \\
u_{4} & u_{2}
\end{array}\right)=\left(\begin{array}{ll}
y_{3} & y_{1} \\
y_{4} & y_{2}
\end{array}\right)
$$

in which

$$
\begin{aligned}
& y_{3}=\frac{\partial u_{4}}{\partial x}-i \frac{\partial u_{4}}{\partial y}+\frac{\partial u_{3}}{\partial z}, \\
& y_{4}=\frac{\partial u_{3}}{\partial x}+i \frac{\partial u_{3}}{\partial y}-\frac{\partial u_{4}}{\partial z}, \\
& y_{1}=\frac{\partial u_{2}}{\partial x}-i \frac{\partial u_{2}}{\partial y}+\frac{\partial u_{1}}{\partial z}, \\
& y_{2}=\frac{\partial u_{1}}{\partial x}+i \frac{\partial u_{1}}{\partial y}-\frac{\partial u_{2}}{\partial z} .
\end{aligned}
$$

Substituting (68) and (69) in (67) results in a matrix presentation for the Maxwell Eqs. (A29) and (A32):

$$
\begin{array}{r}
\frac{\partial u_{4}}{\partial x}-i \frac{\partial u_{4}}{\partial y}+\frac{\partial u_{3}}{\partial z}-\nabla \cdot \Phi=\frac{1}{c} \frac{\partial u_{3}}{\partial t}, \\
\frac{\partial u_{3}}{\partial x}+i \frac{\partial u_{3}}{\partial y}-\frac{\partial u_{4}}{\partial z}=\frac{1}{c} \frac{\partial u_{4}}{\partial t}, \\
\frac{\partial u_{2}}{\partial x}-i \frac{\partial u_{2}}{\partial y}+\frac{\partial u_{1}}{\partial z}=\frac{1}{c} \frac{\partial u_{1}}{\partial t}, \\
\frac{\partial u_{1}}{\partial x}+i \frac{\partial u_{1}}{\partial y}-\frac{\partial u_{2}}{\partial z}-\nabla \cdot \Phi=\frac{1}{c} \frac{\partial u_{2}}{\partial t} .
\end{array}
$$

The Maxwell Eqs. (70) demonstrate an asymmetry in relation to the spatial charge density which is countered by adding and subtracting Eqs. (70a), (70c) and (70b), (70d), respectively:

$$
\begin{aligned}
& \frac{\partial\left(u_{2}+u_{4}\right)}{\partial x}-i \frac{\partial\left(u_{2}+u_{4}\right)}{\partial y}+\frac{\partial\left(u_{1}+u_{3}\right)}{\partial z}-\nabla \Phi=\frac{\partial\left(u_{1}+u_{3}\right)}{\partial c t}, \\
& \frac{\partial\left(u_{1}+u_{3}\right)}{\partial x}+i \frac{\partial\left(u_{1}+u_{3}\right)}{\partial y}-\frac{\partial\left(u_{2}+u_{4}\right)}{\partial z}-\nabla \Phi=\frac{\partial\left(u_{2}+u_{4}\right)}{\partial c t}, \\
& \frac{\partial\left(u_{2}-u_{4}\right)}{\partial x}-i \frac{\partial\left(u_{2}-u_{4}\right)}{\partial y}+\frac{\partial\left(u_{1}-u_{3}\right)}{\partial z}+\nabla \Phi=\frac{\partial\left(u_{1}-u_{3}\right)}{\partial c t}, \\
& \frac{\partial\left(u_{1}-u_{3}\right)}{\partial x}+i \frac{\partial\left(u_{1}-u_{3}\right)}{\partial y}-\frac{\partial\left(u_{2}-u_{4}\right)}{\partial z}-\nabla \Phi=\frac{\partial\left(u_{2}-u_{4}\right)}{\partial c t} .
\end{aligned}
$$

In a charge-free region it follows from (A3), (A30), and (A31) that $\Phi(x, t)$ is divergence-free, and the matrix presentation (71) for the Maxwell equations equals the Dirac equation for elementary particles (e.g., photons) with zero rest mass (Ref. 17, pp. $351,379)$. In a charge-free region, $\nabla \cdot \Phi=0$ and the Maxwell Eqs. (71) can be rewritten as 


$$
\begin{gathered}
\frac{\partial\left(u_{2}+u_{4}\right)}{\partial x}-i \frac{\partial\left(u_{2}+u_{4}\right)}{\partial y}+\frac{\partial\left(u_{1}+u_{3}\right)}{\partial z}=\frac{1}{c} \frac{\partial\left(u_{1}+u_{3}\right)}{\partial t} \\
\frac{\partial\left(u_{1}+u_{3}\right)}{\partial x}+i \frac{\partial\left(u_{1}+u_{3}\right)}{\partial y}-\frac{\partial\left(u_{2}+u_{4}\right)}{\partial z}=\frac{1}{c} \frac{\partial\left(u_{2}+u_{4}\right)}{\partial t} \\
\frac{\partial\left(u_{2}-u_{4}\right)}{\partial x}-i \frac{\partial\left(u_{2}-u_{4}\right)}{\partial y}+\frac{\partial\left(u_{1}-u_{3}\right)}{\partial z}=\frac{1}{c} \frac{\partial\left(u_{1}-u_{3}\right)}{\partial t} \\
\frac{\partial\left(u_{1}-u_{3}\right)}{\partial x}+i \frac{\partial\left(u_{1}-u_{3}\right)}{\partial y}-\frac{\partial\left(u_{2}-u_{4}\right)}{\partial z}=\frac{1}{c} \frac{\partial\left(u_{2}-u_{4}\right)}{\partial t} .
\end{gathered}
$$

The corresponding spinor $\bar{U}_{C}$ is given by (64):

$$
U_{C}=\left(\begin{array}{l}
u_{1}+u_{3} \\
u_{2}+u_{4} \\
u_{1}-u_{3} \\
u_{2}-u_{4}
\end{array}\right)=\left(\begin{array}{c}
\Phi_{X}-i \Phi_{Y}+\Phi_{Z} \\
\Phi_{X}+i \Phi_{Y}-\Phi_{Z} \\
\Phi_{X}-i \Phi_{Y}-\Phi_{Z} \\
-\Phi_{X}-i \Phi_{Y}-\Phi_{Z}
\end{array}\right)
$$

Because mass is a relativistic effect of (confined) energy, it is reasonable to couple the coordinate system of the electromagnetic energy confinement relativistically to the coordinate system of the observer to introduce the relativistic effects of confined energy, observed as a finite rest mass, into the continuity Eq. (57) or its equivalent, the Maxwell Eqs. (72). It is taken into account that the Maxwell Eqs. (72) describe in principle particles with a zero rest mass (e.g., photons). To derive from (72) the Dirac equation, which describes elementary particles with a finite rest mass, a simultaneous particle-antiparticle combination is chosen which particle pair has to satisfy the Maxwell Eqs. (72). This was done before by the relativistic derivation of the Schrodinger Eq. (56) from the continuity Eq. (42) and in a comparable way in the following by the derivation of Newton's law (111) from the relativistic radiation pressure derived from (108).

This will be realized by defining the relativistic vector wave function $\Phi(x, t)$ in (13), describing the electromagnetic field configuration, which is split into two parts:

$$
\Phi=\Phi_{1}+\Phi_{2} .
$$

Equation (74) applies to a monochromatic electromagnetic wave with frequency $\omega_{0}$, which is observed by an observer with a relative velocity $\nabla_{G}$ with respect to the confined electromagnetic wave. In this relation the function $\Phi$ consists of an elementary function $\Phi_{1}$ and a relativistic function $\Phi_{2}$ which describes the transformed relativistic part. For the elementary function $\Phi_{1}$ we have the relation

$$
\begin{gathered}
\Phi_{1}=\frac{\gamma \Phi_{R}}{8}\left(e^{i \alpha}+e^{-i \alpha}\right)\left(e^{i \beta}+e^{-i \beta}\right) \mathbf{e}_{H} \\
-\frac{i \gamma \Phi_{R}}{8}\left(e^{i \alpha}-e^{-i \alpha}\right)\left(e^{i \beta}-e^{-i \beta}\right) \mathbf{e}_{E}
\end{gathered}
$$

in which $\Phi_{R}\left(x^{\prime}, t^{\prime}\right)$ is a real scalar wave function representing the position and time-dependent amplitude of the electromagnetic wave confinement, and $\beta$ and $\alpha$ are represented by (28) and (29), respectively. The vectors $e_{H}$ and $e_{E}$ are unit vectors in the direction of the magnetic field intensity and the electric field intensity, respectively. For the relativistic function $\Phi_{2}$ we have the relation

$$
\begin{gathered}
\Phi_{2}=\frac{i \gamma \Phi_{R}}{8 c}\left(e^{i \alpha}+e^{-i \alpha}\right)\left(e^{i \beta}+e^{-i \beta}\right) \mathbf{v}_{G} \times \mathbf{e}_{H} \\
+\frac{\gamma \Phi_{R}}{8 c}\left(e^{i \alpha}-e^{-i \alpha}\right)\left(e^{i \beta}-e^{-i \beta}\right) \mathbf{v}_{G} \times \mathbf{e}_{E} .
\end{gathered}
$$

Assuming a monochromatic electromagnetic wave packet with rest frequency $\omega_{0}$, for the electric field intensity $\mathbf{E}\left(\mathbf{x}^{\prime}, t^{\prime}\right)$ according to (22) in the coordinate system of an observer moving at a relative velocity $\mathbf{v}_{G}$ with respect to the confined wave, the following relation applies for the observed electric field intensity:

$$
\mathbf{E}^{\prime}\left(\mathbf{x}^{\prime}, t^{\prime}\right)=\frac{\gamma c \Phi_{R}}{(2 \epsilon)^{1 / 2}}\left[e_{E} \sin (\alpha) \sin (\beta)+\frac{\nabla_{G} \times \mathbf{e}_{H}}{c} \cos (\alpha) \cos (\beta)\right)
$$

and according to (23) for the observed magnetic field intensity:

$$
\begin{aligned}
& \mathbf{H}\left(\mathbf{x}^{\prime}, t^{\prime}\right) \\
& =\frac{\gamma \Phi_{R}}{\mu(2 \epsilon)^{1 / 2}}\left[\mathbf{e}_{H} \cos (\alpha) \cos (\beta)-\frac{\mathbf{v}_{G} \times \mathbf{e}_{E}}{c} \sin (\alpha) \sin (\beta)\right)
\end{aligned}
$$

When the observer is at rest, relative to the electromagnetic field confinement, using (28) and (29), Eq. (77) reduces to

$$
\mathbf{E}(\mathbf{x}, t)=\frac{c \Phi_{R}(\mathbf{x}, t)}{(2 \epsilon)^{1 / 2}} \sin (\mathbf{k} \cdot \mathbf{x}) \sin \left(\omega_{0} t\right) \mathbf{e}_{\mathbf{E}}
$$

and a magnetic field intensity is observed equal to

$$
\mathbf{H}(\mathbf{x}, t)=\frac{\Phi_{R}(\mathbf{x}, t)}{\mu(2 \epsilon)^{1 / 2}} \cos (\mathbf{k} \cdot \mathbf{x}) \cos \left(\omega_{0} t\right) \mathbf{e}_{H} .
$$

To split the spinor (73) into two parts, describing the parts $+i \omega$ and $-i \omega$, respectively, Eq. (74) is rewritten into

$$
\Phi=\Phi_{A}+\Phi_{B}
$$

where the vector wave function $\Phi_{A}$ yields the relation 


$$
\Phi_{A}=\frac{\gamma \Phi_{R}}{4}\left[1+\frac{i \nabla_{G}}{c} \times\right]\left[\cos (\alpha) e_{H}+\sin (\alpha) e_{E}\right] e^{i \beta}=\Phi_{R A} e^{i \beta}
$$

and the vector wave function $\Phi_{B}$ yields

$$
\Phi_{B}=\frac{\gamma \Phi_{R}}{4}\left[1+\frac{i \mathbf{v}_{G}}{c} \times\right]\left[\cos (\alpha) \mathbf{e}_{H}-\sin (\alpha) \mathbf{e}_{E}\right] e^{-i \beta}=\Phi_{R B} e^{-i \beta}
$$

where $\Phi_{R A}$ and $\Phi_{R B}$ are real vector functions for the terms $e^{i \beta}$ and $e^{-i \beta}$. From (82) and (83) it follows that the complex vector wave function $\Phi$ can be divided into terms of $e^{i \beta}$ and $e^{-i \beta}$, which has already been reported by Pauli. ${ }^{(16)}$ For the electromagnetic mass distribution of a monochromatic (self-) confined electromagnetic wave it follows from (14) and (81) that

$$
\rho_{E M}(\mathbf{x}, t)=\left(\Phi_{A}+\Phi_{B}\right) \cdot\left(\Phi_{A}^{*}+\Phi_{B}^{*}\right)
$$

An $x-y-z$ coordinate system is introduced, which is rotated in such a way that the velocity $\nabla_{G}$ of the observer relative to the confinement is along the $z$-axis. A linear superposition of confined waves propagating along the $z$-axis is considered, describing a monochromatic electromagnetic wave packet. A monochromatic wave packet around frequency $\omega_{0}$ is considered, which is determined by

$$
\Phi\left(\mathbf{x}^{\prime}, t^{\prime}\right)=\int_{k_{0}}^{k_{0}+\Delta t} \int_{\omega_{0}-\Delta \omega}^{\omega_{0}+\Delta \omega} \phi\left(\mathbf{k}^{\prime}, \omega^{\prime}\right) \exp \left[i\left(\mathbf{k}^{\prime} \cdot \mathbf{x}^{\prime}+\omega^{\prime} t^{\prime}\right)\right] d \mathbf{k}^{\prime} d \omega^{\prime}
$$

in which $\Phi(x, t)$ presents the scalar components in (82) and (83) and $\Delta \omega / \omega_{0} \ll 1$ as well as $\Delta k / k_{0} \ll 1$. The vector function $\Phi_{z}$ represents the part of the vector wave function describing waves propagating only in the $z$-direction. From (82) and (83) it follows for the $x$ and $y$ components, under the restriction that the electric field intensity is oriented along the $x$-axis,

$$
\begin{aligned}
& \Phi_{A Z}=\frac{\gamma \Phi_{R X}}{4}\left(1+\frac{i \mathbf{v}_{G}}{c} \times\right]\left[\cos (\alpha) \mathbf{e}_{Y}+\sin (\alpha) \mathbf{e}_{X}\right] e^{i \beta}=\Phi_{R A X} e^{i \beta} \\
& \Phi_{B Z}=\frac{\gamma \Phi_{R X}}{4}\left[1+\frac{i \mathbf{v}_{G}}{c} \times\right]\left[\cos (\alpha) \mathbf{e}_{Y}-\sin (\alpha) \mathbf{e}_{X}\right] e^{-i \beta}=\Phi_{R B X} e^{-i \beta}
\end{aligned}
$$

The vector wave function, describing the confined electromagnetic wave packet equals

$$
\Phi\left(\mathbf{x}^{\prime}, t^{\prime}\right)=\Phi_{R A X} e^{i \beta}+\Phi_{R B X} e^{-i \beta} .
$$

The observer is moving in a positive direction along the $z$-axis. The coordinate system is oriented in such a way that $e_{X} \times e_{Y}=$ e. This results in a relation for the relativistic terms in (82) and (83), which equals $v_{G} \times e_{H}=-v e_{x}$ and $v_{G} \times e_{E}=v e_{Y}$. Substitution of (82) and (83) in (87) and using (73) and (A3) results in

$$
U_{C}=\frac{\gamma \Phi_{R}\left(x^{\prime}, t^{\prime}\right)}{4}\left[\begin{array}{c}
-i\left[1+\frac{v}{c}\right] e^{i(\beta+\alpha)}-i\left[1+\frac{v}{c}\right] e^{-i(\beta+\alpha)} \\
i\left[1-\frac{v}{c}\right] e^{i(\beta-\alpha)}+i\left[1-\frac{v}{c}\right] e^{-i(\beta-\alpha)} \\
-i\left[1+\frac{v}{c}\right] e^{i(\beta+\alpha)}-i\left[1+\frac{v}{c}\right] e^{-i(\beta+\alpha)} \\
-i\left[1-\frac{v}{c}\right] e^{i(\beta-\alpha)}-i\left[1-\frac{v}{c}\right] e^{-i(\beta-\alpha)}
\end{array}\right]
$$

The $A$ part in (82) describes the $e^{i \beta}$ terms and the $B$ part in (83) describes the $e^{-i \beta}$ terms for the two constituent parts. To describe a particle-antiparticle combination, the spinor in (73) is split into two parts, presented by (89):

$$
U_{c}=\left(\begin{array}{c}
u_{1}+u_{3} \\
u_{2}+u_{4} \\
u_{1}-u_{3} \\
u_{2}-u_{4}
\end{array}\right)=\overline{V^{*}}+\overline{V^{*}}=\left[\begin{array}{l}
\tilde{u}_{1} \\
\tilde{u}_{2} \\
\tilde{u}_{3} \\
\tilde{u}_{4}
\end{array}\right]+\left[\begin{array}{c}
\tilde{u}_{3} \\
-\tilde{u}_{4} \\
\tilde{u}_{1} \\
-\tilde{u}_{2}
\end{array}\right) .
$$

Because new spinor components are created that essentially differ from the original spinor components in (64), these components are indicated by $\tilde{u}$. Combining (73) and (88) defines the way to split up both spinors:

$U_{c}=\nabla^{*}+\nabla^{-}$

$$
=\left(\begin{array}{c}
\left(\Phi_{A R X}-i \Phi_{A R Y}+\Phi_{A R Z}\right) e^{i \beta} \\
\left(\Phi_{A R X}+i \Phi_{A R Y}-\Phi_{A R Z}\right) e^{i \beta} \\
\left(\Phi_{B R X}-i \Phi_{B R Y}-\Phi_{B R Z}\right) e^{-i \beta} \\
\left(-\Phi_{B R X}-i \Phi_{B R Y}-\Phi_{B R Z}\right) e^{-i \beta}
\end{array}\right)+\left(\begin{array}{l}
\left(\Phi_{B R X}-i \Phi_{B R Y}+\Phi_{B R Z}\right) e^{-i \beta} \\
\left(\Phi_{B R X}+i \Phi_{B R Y}-\Phi_{B R Z}\right) e^{-i \beta} \\
\left(\Phi_{A R X}-i \Phi_{A R Y}-\Phi_{A R Z}\right) e^{i \beta} \\
\left(-\Phi_{A R X}-i \Phi_{A R Y}-\Phi_{A R Z}\right) e^{i \beta}
\end{array}\right)
$$

The spinor (90) is split into two parts, describing, respectively, a particle-antiparticle presentation which results in 


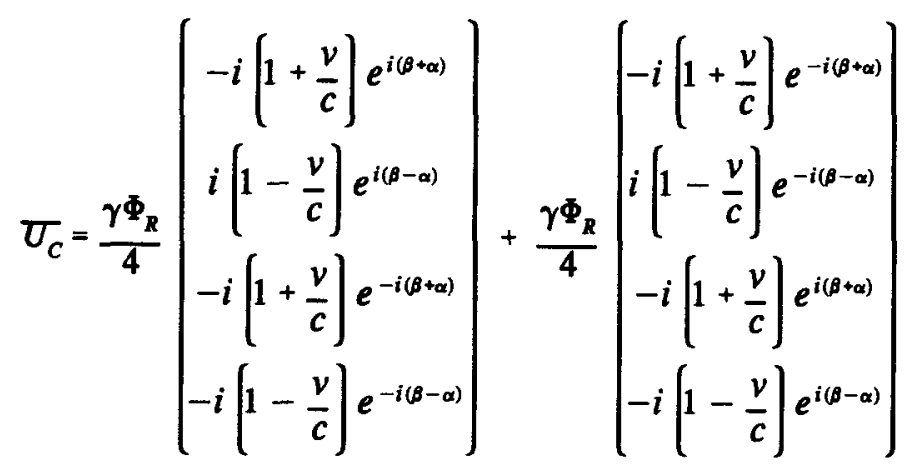

By splitting the spinor ${ }^{(15)}$ into two antisymmetric parts [antisymmetric in the arguments $\alpha$ and $\beta$, presented by (28) and (29)], the Maxwell equations also have to be split into the antisymmetric corresponding parts, with the restriction that the superposition of both spinors and corresponding equations equals the Maxwell Eqs. (72). The Maxwell Eqs. (72) are split in the following way. Equations (72a) and (72b) are split into the Dirac equation with positive time derivatives:

$$
\begin{aligned}
& \frac{\partial\left(\tilde{u}_{4}\right)}{\partial x}-i \frac{\partial\left(\tilde{u}_{4}\right)}{\partial y}+\frac{\partial\left(\tilde{u}_{3}\right)}{\partial z}=\frac{1}{c} \frac{\partial\left(\tilde{u}_{1}\right)}{\partial t} \\
& \frac{\partial\left(\tilde{u}_{2}\right)}{\partial x}-i \frac{\partial\left(\tilde{u}_{2}\right)}{\partial y}+\frac{\partial\left(\tilde{u}_{1}\right)}{\partial z}=\frac{1}{c} \frac{\partial\left(\tilde{u}_{3}\right)}{\partial t} \\
& \frac{\partial\left(\tilde{u}_{3}\right)}{\partial x}+i \frac{\partial\left(\tilde{u}_{3}\right)}{\partial y}-\frac{\partial\left(\tilde{u}_{4}\right)}{\partial z}=\frac{1}{c} \frac{\partial\left(\tilde{u}_{2}\right)}{\partial t} \\
& \frac{\partial\left(\tilde{u}_{1}\right)}{\partial x}+i \frac{\partial\left(\tilde{u}_{1}\right)}{\partial y}-\frac{\partial\left(\tilde{u}_{2}\right)}{\partial z}=\frac{1}{c} \frac{\partial\left(\tilde{u}_{4}\right)}{\partial t}
\end{aligned}
$$

The Maxwell Eqs. (72c) and (72d) are split into the Dirac equation with negative time derivatives:

$$
\begin{aligned}
& \frac{\partial\left(\tilde{u}_{4}\right)}{\partial x}-i \frac{\partial\left(\tilde{u}_{4}\right)}{\partial y}+\frac{\partial\left(\tilde{u}_{3}\right)}{\partial z}=-\frac{1}{c} \frac{\partial\left(\tilde{u}_{1}\right)}{\partial t} \\
& \frac{\partial\left(\tilde{u}_{2}\right)}{\partial x}-i \frac{\partial\left(\tilde{u}_{2}\right)}{\partial y}+\frac{\partial\left(\tilde{u}_{1}\right)}{\partial z}=-\frac{1}{c} \frac{\partial\left(\tilde{u}_{3}\right)}{\partial t} \\
& \frac{\partial\left(\tilde{u}_{3}\right)}{\partial x}+i \frac{\partial\left(\tilde{u}_{3}\right)}{\partial y}-\frac{\partial\left(\tilde{u}_{4}\right)}{\partial z}=-\frac{1}{c} \frac{\partial\left(\tilde{u}_{2}\right)}{\partial t} \\
& \frac{\partial\left(\tilde{u}_{1}\right)}{\partial x}+i \frac{\partial\left(\tilde{u}_{1}\right)}{\partial y}-\frac{\partial\left(\tilde{u}_{2}\right)}{\partial z}=-\frac{1}{c} \frac{\partial\left(\tilde{u}_{4}\right)}{\partial t}
\end{aligned}
$$

$$
\begin{aligned}
& -i \hbar \frac{\partial e^{i \beta}}{\partial t}=\gamma \omega_{0} \hbar e^{i \beta}=W e^{i \beta} \\
& -i \hbar \frac{\partial e^{i \beta}}{\partial z}=\frac{\gamma \omega_{0} \hbar v_{z} e^{i \beta}}{c^{2}}=\frac{W v_{z}}{c^{2}} e^{i \beta}=p_{z} e^{i \beta} \\
& -i \hbar \frac{\partial e^{i \alpha}}{\partial t}=\frac{\gamma \omega_{0} \hbar v_{z} e^{i \alpha}}{c}=\frac{W v_{z}}{c} e^{i \alpha}=c p_{z} e^{i \alpha} \\
& -i \hbar \frac{\partial e^{i \alpha}}{\partial z}=\frac{\gamma \omega_{0} \hbar e^{i \alpha}}{c}=\frac{W}{c} e^{i \alpha} \\
& \frac{\partial e^{i \beta}}{\partial x}=\frac{\partial e^{i \alpha}}{\partial x}=\frac{\partial e^{i \beta}}{\partial y}=\frac{\partial e^{i \alpha}}{\partial y}=0 .
\end{aligned}
$$

Restricted to the conditions of propagation of the (confined) waves in the $z$-direction, it follows from (94)

$$
\begin{aligned}
& -i \hbar \frac{\partial e^{i(\beta+\alpha)}}{\partial t}=W\left(1+\frac{v_{z}}{c}\right) e^{i(\beta+\alpha)} \\
& -i \hbar \frac{\partial e^{i(\beta-\alpha)}}{\partial t}=W\left[1-\frac{v_{z}}{c}\right) e^{i(\beta-\alpha)} \\
& -i \hbar \frac{\partial e^{-i(\beta+\alpha)}}{\partial t}=-W\left[1+\frac{v_{z}}{c}\right] e^{-i(\beta+\alpha)} \\
& -i \hbar \frac{\partial e^{-i(\beta-\alpha)}}{\partial t}=-W\left[1-\frac{v_{z}}{c}\right] e^{-i(\beta-\alpha)}
\end{aligned}
$$

When the observer is at rest relative to the electromagnetic confinement, the time derivative of the spinor $\bar{\nabla}^{+}$is considered:

$\frac{4}{c} \frac{\partial \overline{V^{*}}}{\partial t^{\prime}}$

$$
=\left(\begin{array}{c}
-\frac{i}{c} \frac{\partial\left(\Phi_{R} e^{i(\beta+\alpha)}\right)}{\partial t} \\
\frac{i}{c} \frac{\partial\left(\Phi_{R} e^{i(\beta-\alpha)}\right)}{\partial t} \\
-\frac{i}{c} \frac{\partial\left(\Phi_{R} e^{-i(\beta+\alpha)}\right)}{\partial t} \\
-\frac{i}{c} \frac{\partial\left(\Phi_{R} e^{-i(\beta-\alpha)}\right)}{\partial t}
\end{array}\right)=\left(\begin{array}{c}
-i\left(\frac{i m_{E} c}{\hbar_{E}} \Phi_{R}+\frac{1}{c} \frac{\partial \Phi_{R}}{\partial t}\right) e^{i(\beta+\alpha)} \\
i\left(\frac{i m_{E} c}{\hbar_{E}} \Phi_{R}+\frac{1}{c} \frac{\partial \Phi_{R}}{\partial t}\right) e^{i(\beta-\alpha)} \\
-i\left(\frac{-i m_{E} c}{\hbar_{E}} \Phi_{R}+\frac{1}{c} \frac{\partial \Phi_{R}}{\partial t}\right) e^{-i(\beta+\alpha)} \\
-i\left(\frac{-i m_{E} c}{\hbar_{E}} \Phi_{R}+\frac{1}{c} \frac{\partial \Phi_{R}}{\partial t}\right) e^{-i(\beta-\alpha)}
\end{array}\right)
$$

Restricted to (confined) electromagnetic waves propagating along the $z$-axis, it follows from (21), (28), and (29) that the relations for the time and spatial derivatives of the spinor components $e^{i \beta}$ and $e^{j \alpha}$ in (91) are in which the notation $m_{E}$ denotes an electromagnetic mass. In stationary conditions of the confinement, $\partial_{t} \Phi_{R}=0$, on which condition the substitution of (96) in (93) results in the stationary 
Maxwell equation for confined monochromatic radiation with rest frequency $\omega_{0}=m_{E} c^{2} / \hbar_{E}$ :

$$
\begin{aligned}
& \frac{\partial \tilde{u}_{4}}{\partial x}-i \frac{\partial \tilde{u}_{4}}{\partial y}+\frac{\partial \tilde{u}_{3}}{\partial z}+\frac{i m_{E} c}{\hbar_{E}} \tilde{u}_{1}=0(\mathrm{cA}) \\
& \frac{\partial \tilde{u}_{3}}{\partial x}+i \frac{\partial \tilde{u}_{3}}{\partial y}-\frac{\partial \tilde{u}_{4}}{\partial z}+\frac{i m_{E} c}{\hbar_{E}} \tilde{u}_{2}=0(\mathrm{dA}) \\
& \frac{\partial \tilde{u}_{2}}{\partial x}-i \frac{\partial \tilde{u}_{2}}{\partial y}-\frac{\partial \tilde{u}_{1}}{\partial z}-\frac{i m_{E} c}{\hbar_{E}} \tilde{u}_{3}=0(\mathrm{cB}) \\
& \frac{\partial \tilde{u}_{1}}{\partial x}+i \frac{\partial \tilde{u}_{1}}{\partial y}-\frac{\partial \tilde{u}_{2}}{\partial z}-\frac{i m_{E} c}{\hbar_{E}} \tilde{u}_{4}=0 .(\mathrm{dB})
\end{aligned}
$$

The nonstationary Maxwell equations for confined monochromatic radiation follow from (96), in which the time derivative is related to the mode fluctuation of the (confined) electromagnetic wave and is expressed by $\partial_{t} \Phi_{R}$, which leads to the restriction that the time derivatives in (98) are not operating on the rest frequency $\omega_{0}$ but only on the relative slow fluctuations expressed by $\partial_{t} \Phi_{R^{*}}$

$$
\begin{aligned}
& \frac{\partial \tilde{u}_{4}}{\partial x}-i \frac{\partial \tilde{u}_{4}}{\partial y}+\frac{\partial \tilde{u}_{3}}{\partial z}+\frac{i m_{E} c}{\hbar_{E}} \tilde{u}_{1}+\frac{\partial \tilde{u}_{1}}{\partial t}=0(\mathrm{cA}) \\
& \frac{\partial \tilde{u}_{3}}{\partial x}+i \frac{\partial \tilde{u}_{3}}{\partial y}-\frac{\partial \tilde{u}_{4}}{\partial z}+\frac{i m_{E} c}{\hbar_{E}} \tilde{u}_{2}+\frac{\partial \tilde{u}_{2}}{\partial t}=0(\mathrm{dA}) \\
& \frac{\partial \tilde{u}_{2}}{\partial x}-i \frac{\partial \tilde{u}_{2}}{\partial y}-\frac{\partial \tilde{u}_{1}}{\partial z}-\frac{i m_{E} c}{\hbar_{E}} \tilde{u}_{3}+\frac{\partial \tilde{u}_{3}}{\partial t}=0(\mathrm{cB}) \\
& \frac{\partial \tilde{u}_{1}}{\partial x}+i \frac{\partial \tilde{u}_{1}}{\partial y}-\frac{\partial \tilde{u}_{2}}{\partial z}-\frac{i m_{E} c}{\hbar_{E}} \tilde{u}_{4}+\frac{\partial \tilde{u}_{4}}{\partial t}=0 .(\mathrm{dB})
\end{aligned}
$$

Within the scope of an electromagnetic model of matter, a particle-antiparticle combination is described by the superposition of both Maxwell Eqs. (92) and (93), operating on the superposition of the spinors $\bar{V}^{+}$and $\bar{V}^{-}$, which equals the Maxwell Eq. (72). The following matrices are introduced:

$$
\bar{\alpha}=\left(\begin{array}{ll}
0 & \sigma \\
\sigma & 0
\end{array}\right), \quad \beta=\left[\begin{array}{cc}
\delta_{A B} & 0 \\
0 & -\delta_{A B}
\end{array}\right)
$$

Using (99) and (89), $\bar{V}^{-}+\bar{V}^{+}=\bar{U}_{c}$, the Maxwell Eqs. (72) are split into Eqs. (92), which are presented as

$$
-\frac{1}{c} \frac{\partial \overline{V^{-}}}{\partial t}+\bar{\alpha} \cdot \nabla \nabla^{=}+\frac{i m_{E} c}{\hbar_{E}} \beta \nabla^{=}=0
$$

and (93), which equals

$$
\frac{1}{c} \frac{\partial \overline{V^{*}}}{\partial t}+\bar{\alpha} \cdot \nabla \nabla^{*}+\frac{i m_{E} c}{\hbar_{E}} \beta \nabla^{*}=0
$$

in which $m_{E}$ is the total electromagnetic mass of a particleantiparticle combination, originating from an electromagnetic confinement. The quantum mechanical (relativistic) Dirac equation (Ref. 17, p. 351 ) is presented by

$$
\frac{1}{c} \frac{\partial \Psi}{\partial t}+\bar{\alpha} \cdot \nabla \bar{\Psi}+\frac{i m c}{\hbar} \bar{\Psi} \bar{\Psi}=0 .
$$

The summation of (100) and (101) operating on the spinor $\bar{V}=$ $\bar{V}^{-}+\bar{V}^{+}$equals the Maxwell Eq. (72) operating on an electromagnetic (monochromatic) confinement with rest frequency $\omega_{0}=$ $M_{E} c^{2} / \hbar_{E}$

\section{AUTOCONFINED ELECTROMAGNETIC ENTITIES (AEONs)}

The description of electromagnetism employed in this section refers to an earlier idea of Lorentz, namely, the basic idea that the observed relativistic effects of space and time transformations are essentially based on electromagnetic transformations, which in this theory are considered as the basic fundamentals of space and time. This implies that the idea of a fundamental aether in this section is not principally excluded. The theory, however, requires that the rest mass of the hypothetical aether is zero, and that the observed energy has to be described in terms of an aether tension due to an electromagnetic effect, while this tension is represented by a specific local mass density due to an electromagnetic energy density. This implies that in absolute empty space (without the presence of any energy) the aether does not physically exist, but is created by the presence of electromagnetic energy.

The theory requires that the observed aether phenomena do not contradict special or general relativity. This requirement can only be fulfilled by the assumption that observers are essentially made of AEON combinations, with the observer's own system variables $\Sigma$ in which the time is determined by the rest frequency $\omega_{0}$ of the concerned AEONs and space is prescribed by the corresponding rest wavelengths, indicated by $\mathbf{k}_{0}$ which transform according to (25). Only in that special circumstance, space and time, as discerned by the observer, are fundamentally supported by electromagnetic effects, and the speed of light, described by an electromagnetic effect, will be observed as being independent of the velocity relative to the observer.

The model of an aether that carries the electromagnetic energy transport implies a wave equation that is comparable to an acoustic wave equation in a gas. The relativistic specific local mass of the aether is indicated by $\rho_{\mathrm{EM}}(\mathrm{x}, t)$, and the relativistic local elasticity of the aether is indicated as $\mathscr{E}_{\mathrm{EM}}(\mathrm{x}, t)$. Due to the gradient of the radiation pressure, caused by the local energy density $w$, a volume element $d V$ of electromagnetic energy is accelerated in the direction of the gradient of the radiation 
pressure. This effect is described by the relativistic acoustic aether wave equation which is rather similar to the acoustic wave equation for sound waves in gases, in which the acoustic phenomena are described by the dynamic sound pressure $p_{s}$, which is related to the local dynamic potential energy density of the gas. The acoustic wave equation for sound waves in gases is

$$
\frac{1}{\mathscr{E}_{\mathrm{G}}} \nabla^{2} p_{\mathrm{s}}(\mathbf{x}, t)=\rho_{\mathrm{G}} \frac{\partial^{2} p_{\mathrm{s}}(\mathbf{x}, t)}{\partial t^{2}}
$$

where $\rho_{\mathrm{G}}(\mathbf{x}, t)$ is the local specific mass of the gas, and $\mathscr{E}_{\mathrm{G}}(\mathbf{x}, t)$ is the local elasticity of the gas. By multiplying both sides of Eq. (103) with $-1 / i c \rho$, where $\rho$ is the unit of electric charge density, an electromagnetic equivalent for the local dynamic potential energy density of the gas is obtained by the electric potential $i \varphi / c$. Moving with velocity $v$ relative to the electromagnetic phenomena, the term $i c \rho$ turns out to be the 0 component of the source 4-vector in (5), which transforms due to relativistic effects into $\left(i c \rho^{\prime}\right)(1,-i v / c) \gamma=(i c \rho, \mathbf{j})=j_{a}$, and a Lorentztransformed potential 4-vector is observed which equals $\left(i \varphi^{\prime} / c\right)(1,-i \nabla / c) \gamma=(i \varphi / c, \mathbf{A})=\varphi_{a}$. Using this substitution, Eq. (103) transforms into the relativistic acoustic aether wave equation, in which the term "acoustic" indicates that energy density fluctuations propagate due to an elastic effect:

$$
\frac{1}{\mathscr{E}_{\mathrm{EM}}} \nabla^{2} \varphi_{a}(\mathrm{x}, t)=\rho_{\mathrm{EM}} \frac{\partial^{2} \varphi_{a}(\mathrm{x}, t)}{\partial t^{2}}
$$

The elasticity of electromagnetic radiation is determined by the compression of confined radiation over a small distance and measuring the radiation pressure which has to be counterbalanced during the compression. The quotient of the relative deformation and the applied mechanical pressure is indicated as the elasticity of confined radiation and equals the reciprocal of the energy density $w$. The relativistic specific mass of the aether equals $w / c^{2}$. Substitution of these values in (104) results in

$$
\nabla^{2} \varphi_{a}(\mathbf{x}, t)=\frac{1}{c^{2}} \frac{\partial^{2} \varphi_{a}(\mathbf{x}, t)}{\partial t^{2}}
$$

The material-like treatment of light is in correspondence with Maxwell's theory, because (105) is identical to the electromagnetic source equation in vacuo:

$$
\square \varphi_{a}(\mathbf{x}, t)=\mu j_{a}(\mathbf{x}, t)
$$

where $\square$ is the d'Alembertian operator and equals

$$
=-\partial^{a} \partial_{a}=\frac{1}{c^{2}} \frac{\partial^{2}}{\partial t^{2}}-\left(\frac{\partial^{2}}{\partial x^{2}}+\frac{\partial^{2}}{\partial y^{2}}+\frac{\partial^{2}}{\partial z^{2}}\right) .
$$

The aether model, with the restriction that the rest mass of the aether is zero in the absence of energy, does not violate relativity. When an observer moves relative to an electromagnetic wave, a Lorentz-transformed specific mass and a Lorentztransformed elasticity of the aether are observed, which implies that independent of the velocity of the observer still the relative velocity $c$ of the electromagnetic phenomena is measured. This accords with relativity. This model suggests that in the absence of any energy the aether also disappears, which is in correspondence with Mach's principle that "If there is no matter then there is no geometry." The idea of an electromagnetic aether is also used in the description of the effect that the velocity of electromagnetic waves is lowered in matter. General relativity cannot explain this effect because the concerned mass is much too small to cause measurable space-time curvature. The only possible explanation is to couple the electromagnetic field masses of electrons to the electromagnetic aether mass in (104), while the aether elasticity changes a little due to the electromagnetic coupling of the electron to the atom. This effectively lowers the speed of light in materials. Formula (105) is derived rather to demonstrate that an aether theory does not inevitably contradict Maxwell or relativity than that it is a necessary equation to demonstrate the possibility of AEONs.

An important aspect of AEONs is inertia, or passive gravitational mass. ${ }^{(24,25)}$ To explain inertia a simplified model is used in which radiation is confined, for example, between perfect reflecting mirrors. The radiation pressure on the confining system equals $w / 4$, where $w$ represents the sum of the electromagnetic wave energy density and the confining energy density, and in an inertial system the internal forces counterbalance. During an acceleration a of the system the radiation pressure is transformed. The Poynting 4-vector, describing the confined radiation, is transformed by

$$
{ }^{s} S_{a}^{\prime}={ }^{+} L_{b}^{a}+S_{b}+-L_{b}^{a}-S_{b}
$$

which follows from (A10) to (A15) inclusive and (A51) in the Appendix. During the acceleration, the radiation pressure ${ }^{+} \boldsymbol{P}$ on the confining system towards the acceleration is increased, and the radiation pressure $-\boldsymbol{P}$ on the opposite part is decreased. In a one-dimensional reduction (108) can be written as

$$
s S_{1}^{\prime}=\left(\frac{1+v / c}{1-v / c}\right)+S_{1}+\left(\frac{1-v / c}{1+v / c}\right)-S_{1} .
$$

This results in a difference of the radiation pressure ${ }^{(4,5)}+P$ and - $P$ during the acceleration. The resulting force $F_{\mathrm{R}}$ on the system during the acceleration equals

$$
F_{\mathrm{R}}={ }^{+} F+{ }^{-} F=\left[\left(\frac{1+v / c}{1-v / c}\right]-\left[\frac{1-v / c}{1+v / c}\right]\right] \frac{w \Lambda}{4}
$$

where $\Lambda$ is the surface of the confining system. During the acceleration in a time interval $\Delta t$ the waves travel from one 
mirror a distance $l$ to the opposite mirror. For that reason, the traveling time $\Delta t$ equals $l / c$. At uniform acceleration $a$ the velocity $v$ is increased with $a \Delta t$ during the interval $\Delta t$. Substitution of (77) results in

$$
F_{\mathrm{R}}=\left[\left(\frac{1+a l / c^{2}}{1-a l / c^{2}}\right)-\left(\frac{1-a l / c^{2}}{1+a l / c^{2}}\right)\right] \frac{w \Lambda}{4}
$$

At accelerations $a \ll c^{2} / l$, Eq. (111) is approximately equal to Newton's law $F_{\mathrm{R}}=W a / c^{2}$ in which the passive gravitational mass equals $W / c^{2}$. This implies that the model of confined radiation obeys elementary physical laws and gives a reasonable explanation for elementary material aspects of AEONs.

An important aspect of general relativity, the elementary coupling between passive gravitational mass, presented in (111), and active gravitational mass which is the origin of a gravitational field, is the basic of the description of GEONs ${ }^{(1)}$ (gravitational electromagnetic entities). Equation (111) shows that a gradient in the energy density, opposite to the acceleration, causes passive gravitational mass. Subsequently confined electromagnetic radiation, situated in a gravitational field, will present a gradient of the energy density due to this gravitation, which results in a force opposite to the direction of the gravitational field.

This idea is worked out in a description of a static electromagnetic aether self-confinement in which electromagnetic waves propagate comparable to a cloud of gas in which acoustic waves propagate, described by (105) and (103), respectively, in which the term "acoustic" concerns the propagation of energy density fluctuations due to an elastic effect. Equations (104) and (105) describe electromagnetism as an acoustic 4-vector-oriented phenomenon in a medium with a mass density proportional to the energy density of the confined phenomenon and consequently a rest mass equal to zero, in contradiction to Eq. (103), which describes an acoustic scalar phenomenon in a medium with finite rest mass mainly independent of the energy density of the confined acoustic wave in, for example, a gas cloud. The relativistic effects of the electromagnetic waves propagating in the GEONs are in this model observed as probability waves and described by (56) and (102).

Because gravitational forces will not exclusively lead to GEONs, gravity is in this short illustration treated by a Poisson equation instead of the more valid Einstein-Maxwell equations. The description of the electromagnetic aether self-confinement concerns a static phenomenon due to an extremely strong energy density, which is responsible for the confinement. The treated equations are comparable to the equations concerning static gas clouds in free space and will not describe the confining wave equation itself, but only the stability condition for electromagnetic self-confinement.

A simplified approach to the gravitational field generated by an arbitrary mass distribution has been adopted for gravitational self-confinement. To determine the gravitational field a Poisson equation has been formulated for the gravitational potential $V_{G}(\mathbf{x})$, caused by a mass density distribution $\rho_{M}(\mathbf{x})$ :

$$
\nabla^{2} V_{G}(x)=-4 \pi G \rho_{M}(x)
$$

where $G$ is the gravitational constant in (11). If the mass distribution relates to a point mass $M$, we have $\rho_{M}(\mathbf{x})=M \delta(\mathbf{x})$. The solution for the gravitational potential is then $V_{G}(r)=$ $-G M / r$, where $r$ is the distance related to the point mass $M$. If the electromagnetic mass density, defined in (47) and (49), is substituted in (112), this can then be written as

$$
\nabla^{2} V_{G}(\mathbf{x})=-1 / 2 \kappa c^{2} \tilde{\Psi}_{R}^{2}
$$

where $\kappa$ is the coupling constant in (11) and expresses the coupling of an electromagnetic energy density to a gravitational field. The solution of (113) is given by

$$
V_{G}(\mathbf{x})=G \int_{\mathrm{vol}} \frac{\tilde{\mathbf{\Psi}}_{\mathbf{R}}^{2}(\mathbf{y})}{|\mathbf{x}-\mathbf{y}|} d V
$$

The model assumes an equilibrium between the repulsive forces, caused by the gradient of the radiation pressure and the attractive forces generated by the mass of the confined electromagnetic radiation. The occurrence of the repulsive forces in the confined electromagnetic radiation can be explained as follows. In a thought experiment the assumption is made of a doublesided perfectly reflecting mirror of thickness $\Delta x$. A plane electromagnetic wave with energy density $w+\Delta w$ strikes the left side of the mirror, and radiation energy with an energy density $w$ strikes the right side. If the surface area of the mirror is $A$, the resulting force on the mirror is directed to the right and is equal to $A \Delta w$. If the system is situated in a gravitational field with gravitational potential $V_{G}$, a layer thickness of electromagnetic radiation with thickness $\Delta x$ and a surface area $A$ experiences an attractive force equal to $\left(w A \Delta x / c^{2}\right)\left(\partial V_{G} / \partial x\right)$, which concept is in basic correspondence with (A48). In the thought experiment the mirror is now replaced by electromagnetic radiation with energy density $w$. In equilibrium, the rightwards-directed forces, as a result of a gradient in the electromagnetic energy density, will be compensated by the leftwards-directed forces because of a gradient in the gravitational potential. In equilibrium we have the equation

$$
\nabla w=\frac{w}{c^{2}} \nabla V_{G}
$$

from which a simplified stability equation can be derived if the equilibrium relates to the gradient in radial direction (115). The same result follows from (A47). Under these conditions it is possible to give a rough estimate for the required energy density in the case of gravitational self-confinement. By determining the divergence of the left-hand and right-hand terms in (115) and substitution of (113), we obtain 


$$
\nabla^{2} \tilde{\Psi}_{R}=\left(\frac{\nabla \tilde{\Psi}_{R} \cdot \nabla \tilde{\Psi}_{R}}{\tilde{\Psi}_{R}^{2}}-\frac{\kappa \tilde{\Psi}_{R}^{2}}{4}\right) \tilde{\Psi}_{R} .
$$

Relation (116) expresses a condition that must be satisfied by a self-confined electromagnetic wave in radial direction under the influence of a self-generated gravitational field. A particular solution that satisfies (116) is

$$
\tilde{\Psi}_{\mathrm{R}}=\frac{2}{\kappa^{1 / 2}} \frac{1}{r} \approx 1.5 \times 10^{13} \frac{1}{r}
$$

Equation (117) shows the energy density required for gravitational self-confinement. This energy density should be in the order of $10^{73}\left(\mathrm{~J} / \mathrm{m}^{3}\right)$ at the surface of an electromagnetic selfconfinement with, for example, the dimensions of a proton. In view of the small mass of elementary particles, the electromagnetic self-confinement should take place in a very thin energy shell on the surface of the confinement. Because of the local extremely high electromagnetic radiation pressure of $10^{73} \mathrm{~N} / \mathrm{m}^{2}$, a gravitational electromagnetic self-confinement will behave like an extremely hard, nondeformable elementary particle that can only be split in collision experiments.

The values in Table I for the averaged electric field intensity are only comparable with the results from Eq. (117) for low azimuthal quantum number $l \leq 10$.

Table I: Values for the Averaged Electric Field Intensity

\begin{tabular}{lll}
\hline & GEON I & GEON II \\
\hline $\begin{array}{l}\text { Mass }(\mathrm{kg}) \\
\begin{array}{l}\text { Radial coordinate of } \\
\text { active zone (m) }\end{array}\end{array}$ & $10^{39}$ & $10^{39}$ \\
$\begin{array}{l}\text { Spherical harmonic index } \\
l^{*}=[l(l+1)]^{12}\end{array}$ & $1.67 \times 10^{12}$ & $1.67 \times 10^{12}$ \\
$\begin{array}{l}\text { Circular frequency of } \\
\text { emergent radiation }\left(\mathrm{s}^{-1}\right)\end{array}$ & 10 & $8.43 \times 10^{9}$ \\
$\begin{array}{l}\text { Time to collapse } \\
\text { assuming leakage only } \\
\text { rms electric field in most } \\
\text { active region (esu/cm) }\end{array}$ & $6 \times 10^{-4}$ & $5.06 \times 10^{5}$ \\
\hline
\end{tabular}

From Ref. 1.

It follows from Eqs. (117) and (A3) that for a gravitational self-confinement in a spherical shell (azimuthal quantum number $l=0$ ) at a radius of $1.67 \times 10^{12} \mathrm{~m}$ the required averaged field intensity $\tilde{E}=13.5 \times 10^{14} \mathrm{~V} / \mathrm{m}$, which equals in electrostatic units $\tilde{E}=4.5 \times 10^{10} \mathrm{esu} / \mathrm{cm}$ (Table I). Gravitational selfconfinements have already been described by Wheeler ${ }^{(1,26)}$ who introduced GEONs, which describe gravitationally confined radiation in toroidal confinements. Table I presents the values calculated by Wheeler, by solving the Einstein-Maxwell equations for the toroidal gravitational self-confinement.

GEONs at smaller dimensions are quantum objects and need a different mathematical approach. In the simplified example in (116), gravity is only used to confine electromagnetic radiation in the way that gas clouds are confined by their own gravitational field. The mass, however, of an elementary particle is too small for confining gravitationally electromagnetic radiation in a reasonable way. An alternative way of confinement, like "electromagnetostatic" confinement, is required.

In a way identical to the way that GEONs are described by the gravitational equilibrium Eq. (115) or its equivalence (116), EEONs (electromagnetostatic confined electromagnetic entities) are described by the electromagnetostatic equilibrium Eq. (120). The electromagnetostatic balance equation follows from the energy-momentum tensor (8), which can be written as

$$
T^{a b}=\left(\epsilon E_{a} E_{b}+\frac{1}{\mu} B_{a} B_{b}-\delta_{a b} w\right),
$$

where $w$ is the energy density. From (A45) and (A47) in the Appendix it follows that the electromagnetostatic balance equation equals

$$
f^{a}=\partial_{b} T^{a b}=0
$$

which can be written as

$$
\epsilon_{o}[\mathbf{E}(\nabla \cdot \mathbf{E})+(\mathbf{E} \cdot \nabla) \mathbf{E}]+\frac{1}{\mu}[\mathbf{B}(\nabla \cdot \mathbf{B})+(\mathbf{B} \cdot \nabla) \mathbf{B}]=\nabla w
$$

As an example a three-dimensional confinement is chosen in which the vector wave function $\Phi(r, \theta, \varphi, t)$ in (13) or (A3) is presented in spherical coordinates and equals

$$
\Phi(r, \theta, \varphi, t)=\left(\begin{array}{l}
\Phi_{\mathrm{R}} \\
\Phi_{\theta} \\
\Phi_{\varphi}
\end{array}\right]=\left[\frac{\epsilon}{2}\right]^{1 / 2}\left[\begin{array}{c}
i R(r) Y_{m m}(\theta, \varphi) \\
0 \\
R(r) Y_{m m}(\theta, \varphi)
\end{array}\right)
$$

where $R(r) Y_{l m}(\theta, \varphi)$ is a real function. It follows from (135) that in this type of confinement the electric field intensity is oriented along the radial coordinate. The component of $\Phi(r, \theta, \varphi, t)$ along the azimuthal direction equals zero. The magnetic field intensity is oriented along the $\varphi$ direction. The frequency of the confinement is determined by the demand of continuity of the electric and magnetic field intensities so that $n \lambda$ equals the circumference of the confinement. In analogy with the gravitational confinement of electromagnetic radiation, described by Wheeler, ${ }^{(1)}$ an electromagnetostatic confinement of light requires an equilibrium between the radial outwardly pointing radiation pressure and the radial inwardly pointing electromagnetostatic forces. The radial outwardly pointing radiation pressure equals 


$$
-\nabla w=-c^{2} \frac{\partial\left(\Phi \cdot \Phi^{*}\right)}{\partial r}=-\epsilon R(r) Y_{l m}^{2}(\theta, \varphi) \frac{\partial R(r)}{\partial r}
$$

Because in this type of confinement the magnetic field intensity is oriented perpendicular to the radial direction, only the electric field intensity supplies the radial inwardly pointing forces to counterbalance the radial radiation pressure. It follows from Eq. (120) that the radial inwardly pointing electric forces equal

$$
\epsilon\left(E_{\mathrm{R}}(\nabla \cdot \mathbf{E})\right)+(\mathbf{E} \cdot \nabla) E_{\mathrm{R}}=2 \epsilon E_{\mathrm{R}}\left[\frac{\partial E_{\mathrm{R}}}{\partial r}+\frac{E_{\mathrm{R}}}{r}\right]
$$

which can be written as

$$
\begin{aligned}
& \epsilon\left[E_{\mathrm{R}}(\nabla \cdot \mathbf{E})\right]+(\mathbf{E} \cdot \nabla) E_{\mathrm{R}} \\
& =2 \epsilon R(r) Y_{l m}^{2}(\theta, \varphi) \frac{\partial R(r)}{\partial r}+\frac{2}{r} \epsilon R(r)^{2} Y_{l m}^{2}(\theta, \varphi) .
\end{aligned}
$$

An electrodynamic equilibrium exists if the radial inwardly pointing electric forces in (124) counterbalance the radial outwardly pointing radiation pressure. The electromagnetostatic balance equation becomes

$$
\epsilon R(r) Y_{l m}^{2}(\theta, \varphi) \frac{\partial R(r)}{\partial r}+\frac{2}{r} \epsilon R(r)^{2} Y_{l m}^{2}(\theta, \varphi)=0
$$

The electromagnetic equilibrium Eq. (125) can be written as

$$
\frac{\partial R(r)}{\partial r}+\frac{2}{r} R(r)=0
$$

with a particular solution:

$$
R(r)=K / r^{2}
$$

where $K$ is an arbitrary constant. The radial dependence of the function $R(r)$ in (127) for electrostatic self-confinement is different from the solution (117) for gravitational self-confinement. Equation (127) demonstrates that electromagnetostatic confinement is a physical possibility and that the solution (127), which is independent of $\omega$, and $Y_{l m}(\theta, \varphi)$, is in conformity with the solution of the Poisson equation for electric fields. The solutions for the radial-dependent function $R(r)$ are determined by the chosen electromagnetostatic mode. Many aspects have not yet been discussed. But the first treatment of the theoretical possibility of electromagnetostatic self-confinement perhaps will offer possibilities of developing a continuous model of matter. One aspect is that the acoustic model for electromagnetic radiation offers values for the required averaged field intensity at gravitational self-confinements (GEONs) which are reasonably comparable to the solutions found by Wheeler, solving the Einstein-Maxwell equations at low azimuthal quantum number.

\section{CONCLUDING REMARKS}

Because of the high-energy density of self-confined electromagnetic radiation, the radiation pressure is extremely high (depending on the method of self-confinement). For gravitational confinement it varies in magnitude, roughly about $10^{70} \mathrm{~N} / \mathrm{m}^{2}$ as a result of which self-confined electromagnetic radiation behaves as a virtually nondeformable particle in experiments. The model of the self-confinement of electromagnetic radiation presented here is possibly a first step towards a continuous model of matter and may provide a better explanation for several quantum mechanical effects than a particle model of matter.

In this electromagnetic model of matter, the behavior of particles is not described by quantum mechanical probability waves. Particles and the corresponding probability waves are not considered to be complementary aspects of the same object and are unified in such a way that the electromagnetic wave phenomenon itself carries mass, ${ }^{(4)}$, charge, ${ }^{(8)}$ and interaction. ${ }^{(14)}$ Quantum mechanical probability waves are in most treatments considered to be massless and virtual, and by exception only ${ }^{(4,8)}$ are considered to be carriers of charge, mass, ${ }^{(4)}$ or interaction forces. Hence a theory must explain remarkable continuity aspects of particles like tunneling or a continuous charge distribution based on elementary particles, while a characteristic aspect of particles is the discontinuity.

The electromagnetic model ${ }^{(27,23)}$ possibly offers an explanation why electromagnetic radiation should be emitted if an electron drops back to a lower energy level, ${ }^{(2)}$ when the light emitted in this model is regarded as the building material. It offers the possibility of explaining by the effect of spatially generated charge ${ }^{(30)}$ why an electron in a stable orbit around a proton can completely screen this proton electrically, which would be impossible for a point charge like an electron as a particle, if this charge is not moving at infinite velocity. This model may provide an explanation of the fact that an electron in a stable orbit does not emit electromagnetic radiation. ${ }^{(28,30)}$ In it, the Poynting vector of the confined electromagnetic wave is directed along the energy shell, and it is only during the change in energy level that the surface integral of the Poynting vector across the energy shell is positive when the energy drops and negative during a transition to a higher-energy orbit. ${ }^{(31)}$ This could explain why all atoms are excited only by the absorption of electromagnetic radiation and can only drop to a lower energy state when emitting radiation. ${ }^{(27.32)}$

The effect of decreasing the speed of light by matter, like wave propagation in lenses, cannot be explained in terms of general relativity, ${ }^{(33)}$ because the concerned mass is much too small. Considering electromagnetism as an aether-tension effect, (104) and (105) offer the possibility of changing the electromagnetic mass density of the aether or the elasticity by coupling with interacting electrons while not conflicting with Maxwell or relativity. ${ }^{(2,32)}$ The acoustic model of light results in the confinement Eq. (116). The spherical solution (117) for azimuthal quantum number $l=0$ demonstrates a value for the required 
averaged electric field intensity of $4.5 \times 10^{10} \mathrm{esu} / \mathrm{cm}$, which corresponds reasonably with the value of $4.66 \times 10^{10} \mathrm{esu} / \mathrm{cm}$, Table I, calculated by Wheeler ${ }^{(1)}$ by solving the Einstein-Maxwell equations for a GEON with spherical har monic index $l \approx 10$.

The acoustic approach of light (105) possibly will offer a useful method for describing electromagnetostatic modulation to deflect laser beams by electromagnetostatic lenses. Experiments with electrostatically charged microelectrodes with separations comparable to the wavelength of the beam have been done successfully and show the possibility of electromagnetostatic (self-) confinement.

The electromagnetic model of matter is hypothetical. An attempt has been made to prove within the boundaries of classical theories that quantum mechanical probability possibly originates in the relativistic effects of AEONs and that electromagnetic self-confinement is a physical possibility without contradicting Maxwell or relativity. The fact that the Maxwell Eqs. (100) and (101) demonstrate a remarkable correspondence with the Dirac Eq. (102) indicates an electromagnetic origin of matter.

\section{Acknowledgment}

This work has been made possible by the contributions of many. I am indebted to all and my family, in particular.

\section{APPENDIX: CLASSICAL ELECTROMAGNETISM RELATED TO RELATIVTTY AND QUANTUM MECH- ANICS}

This Appendix presents a brief view of classical electromagnetism in relation to special relativity and quantum mechanics. A well-known equation in quantum mechanics is the law of conservation of probability, presented as

$$
\partial w_{P} / \partial t+\nabla \cdot \mathbf{J}=0
$$

where $w_{P}$ is the probability density and equals $\Psi^{*} \Psi$, and $J$ is the probability density flux. The continuity equation for electromagnetic radiation, given in Eq. (42) in the article and (A63) in the Appendix, is presented as a basic equivalent for the law of conservation for probability in which the electromagnetic energy density $w$ is an equivalent for the probability density $w_{p}$ and the Poynting vector $\mathbf{S}$ describing the energy density flux of electromagnetic radiation, turns out to be the equivalent for the probability density flux, presented by $\mathbf{J}$ :

$$
\partial w / \partial t+\nabla \cdot \mathbf{S}=0
$$

By introducing the equivalence of both equations, the law of conservation of probability transforms into the law of conservation of energy. The continuity Eq. (42) is relativistically transformed into (56), which equation has a clear correspondence with the Schrödinger equation for probability waves. The relation between probability and electromagnetic energy density is created by introducing the electromagnetic vector wave functions $\Phi(\mathbf{x}, t)$ and $\Phi^{*}(\mathbf{x}, t)$ in (13). It is important to note that both vector wave functions are complex and have no physical meaning. They are in no way related to a real electric or magnetic field, and physically both hypothetical vector wave functions do not exist. Substitution of (1) and (2) in (13), in the absence of a gravitational field, results in

$$
\Phi(\mathbf{x}, t)=(\epsilon / 2)^{1 / 2}(\mathbf{B}+i \mathbf{E} / c) .
$$

The scalar product of the vector wave functions $\Phi(x, t)$ and $\Phi^{*}(x, t)$ times the square of the velocity of light is a real physical quantity and equals the electromagnetic energy density $w$ :

$$
w(\mathbf{x}, t)=c^{2} \Phi(\mathbf{x}, t) \cdot \Phi^{*}(\mathbf{x}, t)=\frac{1}{2}\left[\frac{B^{2}}{\mu}+\epsilon E^{2}\right] .
$$

This equation is related to the definition of the quantum mechanical probability density $w_{P}$ for which quantity prevails:

$$
w_{P}=\Psi \Psi^{*}
$$

Equations (A4) and (A5) demonstrate an initial correspondence between quantum mechanical probability waves and electromagnetic waves. The electromagnetic energy density flux is presented by the Poynting vector $S$ and equals

$$
\mathbf{S}(\mathbf{x}, t)=i c^{3}\left(\Phi^{*} \times \Phi\right)=\frac{1}{\mu}(\mathbf{E} \times \mathbf{B})
$$

Substituting (A4) and (A6) into (A2) results in an equation for the hypothetical nonexisting vector waves functions $\Phi(x, t)$ and $\Phi *(\mathbf{x}, t)$, which describes a real physical process of the conservation of electromagnetic energy:

$$
\frac{\partial\left(\Phi \cdot \Phi^{*}\right)}{\partial t}+i c \nabla \cdot\left(\Phi^{*} \times \Phi\right)=0
$$

In Sec. 2 the Schrödinger wave function is relativistically derived from Eq. (A7) in a way comparable to Sec. 3 where the relativistic Dirac equation is derived from the same Eq. (A7). This demonstrates a further correspondence between the hypothetical electromagnetic complex wave functions $\Phi(x, t)$ and $\Phi^{*}(\mathrm{x}, t)$ and the quantum mechanical complex probability wave functions $\Psi(x, t)$ and $\Psi^{*}(x, t)$, while both Eqs. (A1) and (A2) describe real physical processes.

To clarify the relativistic derivation of the Schrödinger wave equation from the continuity Eq. (A2), a brief introduction is given in special relativity, which describes the transformations of space, time, and electromagnetism in any inertial system. Because a uniform velocity relative to an object in a coordinate 
system $\Sigma$ can be described as a one-dimensional movement by a rotation of the coordinate system, an explanation is given for a one-dimensional Lorentz transformation along the 1-axis, which is indicated in the following as $x$-axis.

A monochromatic electromagnetic wave with frequency $\omega$ and amplitude $E_{0}$ is considered, confined in an arbitrary system. The considered one dimension (indicated as the $x$-axis) is parallel to the observer's direction of movement. In this description of one dimension a confined electromagnetic wave can be described as

$$
\mathbf{E}(\mathbf{x}, t)=\mathbf{E}_{0} \sin (\omega t) \sin (k x),
$$

where $k=2 \pi / \lambda$, and $\lambda$ is the wavelength of the confined monochromatic wave. When an observer is moving relative to the confined wave (along the $x$-axis), a transformed frequency, wavelength, $x$-coordinate, and $t$-coordinate are observed. It is impossible to transform Eq. (1) by a Lorentz transformation, because every confined wave has to be considered as the superposition of waves propagating away from the observer and waves in opposite direction. For that reason (A8) is written as

$$
\mathbf{E}(\mathbf{x}, t)=\frac{\mathbf{E}_{0}}{2}[\cos (\omega t-k x)-\cos (\omega t+k x)] .
$$

The Lorentz transformation depends on the relative velocity. For waves propagating towards the observer (the velocity of the observer relative to the confining system is $+v$ ) an increased frequency ${ }^{+} \omega$ is measured while waves moving in the opposite direction are observed with a decreased frequency $-\omega$. The shifted frequency ${ }^{+} \omega$ of the waves moving towards the observer is described by the zero component of the wave 4-vector (24), which transforms as follows:

$$
{ }^{+} \omega^{\prime}={ }^{+} k^{0^{\prime}}={ }^{+} L_{a}^{0} k^{a}=\gamma \omega\left(1+\frac{v}{c}\right)
$$

where ${ }^{+} L_{a}^{0}$ is the Lorentz transformation tensor, presented in (A57). The frequency of the waves moving away from the observer is observed as ${ }^{-} \omega^{\prime}$ and equal to

$$
\omega^{\prime}=-k^{0^{\prime}}=-L_{b}^{0} k^{b}=\gamma \omega\left[1-\frac{\nu}{c}\right]
$$

where ${ }^{-} L_{b}^{0}$ is given in (A58). In a comparable way the $x$ component $k$ of the wave 4-vector $k_{1}$ transforms as follows:

$$
{ }^{\dagger} k^{\prime}={ }^{+} k^{1}{ }^{\prime}={ }^{+} L_{b}^{1} k^{b}=\frac{\gamma \omega}{c}\left[1+\frac{\nu}{c}\right]
$$

for the waves moving toward the observer, and as

$$
-k^{\prime}=-k^{1^{\prime}}=-L_{b}^{1} k^{b}=\frac{\gamma \omega}{c}\left[1-\frac{\nu}{c}\right]
$$

for the waves moving in the same direction as the observer. The coordinate $x$, which is the 1 component of the Minkowski 4vector $x^{a}$ will transform thus:

$$
x^{\prime}=L_{a}^{1} x^{a}=\gamma(x-v t)
$$

(The coordinate system $\Sigma^{\prime}$ is connected to the moving observer, and the system $\Sigma$ is connected to the confining system.) The coordinate $i c t$, which is the 0 component of the 4-vector $x^{a}$ will transform thus:

$$
i c t^{\prime}=L_{a}^{0} x^{a}=i c \gamma\left(t-\frac{v x}{c^{2}}\right)
$$

The first part of (A9) presents the waves traveling in the positive $x$-direction and moving in the same direction as the observer. They will be observed with a lowered frequency. The second term in (A9) presents the waves traveling towards the observer; the waves are measured with an increased frequency. The observer perceives the transformed wave

$$
\mathbf{E}^{\prime}\left(\mathbf{x}^{\prime}, t^{\prime}\right)=\frac{\gamma \mathbf{E}_{0}}{2}\left[\cos \left(-\omega^{\prime} t^{\prime}-{ }^{-} k^{\prime} x^{\prime}\right)-\cos \left({ }^{+} \omega^{\prime} t^{\prime}+{ }^{+} k^{\prime} x^{\prime}\right)\right]
$$

Substitution of (A10) to (A13) inclusive in (A16) results in

$$
\begin{aligned}
& \mathbf{E}^{\prime}\left(\mathbf{x}^{\prime}, t^{\prime}\right)=\frac{\gamma \mathbf{E}_{0}}{2}\left[\cos \left\{\gamma \omega\left(1-\frac{\nu}{c}\right) t^{\prime}-\gamma k\left(1-\frac{\nu}{c}\right) x^{\prime}\right\}\right] \\
& -\frac{\gamma \mathbf{E}_{0}}{2}\left[\cos \left\{\gamma \omega\left[1+\frac{\nu}{c}\right) t^{\prime}+\gamma k\left(1+\frac{\nu}{c}\right) x^{\prime}\right\}\right] ;
\end{aligned}
$$

subsequently, (A17) is written in a way comparable to (A8). Using the simple goniometric equation

$$
\cos (p)-\cos (q)=2 \sin \left(\frac{p+q}{2}\right) \sin \left(\frac{q-p}{2}\right)
$$

(A17) can be written as

$$
\mathbf{E}^{\prime}\left(\mathbf{x}^{\prime}, t^{\prime}\right)=\gamma \mathbf{E}_{0} \sin \left(\gamma \omega t^{\prime}+\frac{\gamma k v x^{\prime}}{c}\right) \sin \left(\gamma k x^{\prime}+\frac{\gamma \omega v t^{\prime}}{c}\right) .
$$


Introducing the phases $\alpha$ and $\beta$, (A19) is presented by

$$
\mathbf{E}^{\prime}\left(\mathbf{x}^{\prime}, t^{\prime}\right)=\gamma \mathbf{E}_{0} \sin (\beta) \sin (\alpha) \text {. }
$$

Equation (A20) is comparable to the first part of (27), while $\alpha$ and $\beta$ are presented in a comparable way in (28) and (29) for a one-dimensional wave. The second part of (27) follows from the complete Lorentz transformation for electromagnetic fields, which implies that a part (proportional to $v / c$ ) of an electric field is transformed into a magnetic field, and a part of a magnetic field (proportional to $v / c$ ) is transformed into an electric field due to the transformation of the 4-potential.

The wave in (A19) propagates with an apparent phase velocity (Ref. 3, p. 145), related to the phase $\beta$, indicated as $v_{\beta}$ :

$$
v_{\beta}=\frac{x^{\prime}}{t^{\prime}}=-\frac{\gamma \omega}{\gamma k v / c}
$$

Using the basic relation $k=\omega / c$, (A21) changes into

$$
v_{\beta}=-c^{2} / v \text {. }
$$

In the original article the relative velocity between the observer and the confining system is indicated as the group velocity $v_{G}$ and (A22) is comparable to (30). The negative sign indicates that the observed wave propagates with an apparent phase velocity, inversely proportional to the momentum of the confined wave, towards the observer. From the phase $\beta$ in (A19) it follows that the observed wavelength $\lambda$ equals

$$
\lambda=\frac{2 \pi}{k^{\prime}}=\frac{2 \pi c}{\gamma k v}=\frac{c^{2}}{f \gamma \nu}=\frac{m_{0} c^{2}}{f m \nu}=\frac{W_{0}}{f p} .
$$

Substitution of (21) in (A23) results in

$$
\lambda=h_{\varepsilon} / p .
$$

Equation (A24) shows that the observed wavelength $\lambda$ is inversely proportional to the momentum $p$ of the confined electromagnetic radiation, which is a characteristic phenomenon for probability waves. Substitution of (A14) and (A15) into (A19) yields

$$
\begin{aligned}
\beta & =\left[\gamma \omega t^{\prime}+\frac{\gamma k v x^{\prime}}{c}\right] \\
& =\left[\gamma^{2} \omega\left[t-\frac{v x}{c^{2}}\right]+\frac{\gamma^{2} \omega \nu}{c^{2}}(x-v t)\right]=\omega t
\end{aligned}
$$

This means that the phase $\beta$ is Lorentz-invariant. In a comparable way the phase $\alpha$ equals

$$
\begin{aligned}
\alpha & =\left[\gamma k x^{\prime}+\frac{\gamma \omega v t^{\prime}}{c}\right) \\
& =\left[\gamma^{2} k(x-v t)+\gamma^{2} k v\left[t-\frac{v x}{c^{2}}\right]\right]=k x .
\end{aligned}
$$

Equation (A26) proves that the phase $\alpha$ is also Lorentz-invariant, which is a necessary requirement for a Lorentz transformation.

To derive the Schrodinger Eq. (56) from (A7) relativistically, a Lorentz transformation is required of the Maxwell energy-momentum tensor (8). Substituting (1) and (2) into (4) (in the article), the Maxwell tensor is presented by

$$
F_{a b}=\left|\begin{array}{cccc}
0 & -\frac{i}{c} E_{x} & -\frac{i}{c} E_{y} & -\frac{i}{c} E_{z} \\
\frac{i}{c} E_{x} & 0 & -B_{z} & B_{y} \\
\frac{i}{c} E_{y} & B_{z} & 0 & -B_{x} \\
\frac{i}{c} E_{z} & -B_{y} & B_{x} & 0
\end{array}\right| .
$$

In nonrelativistic units the inhomogeneous Maxwell Eq. (6) becomes

$$
\partial_{b} F_{a b}=-\mu_{0} j_{a}
$$

Substituting (5) in the covariant Maxwell Eq. (A28) and using (A27) results in the inhomogeneous Maxwell equation for $a$ varying from 1 and $b$ varying from 0 to 3 (inclusive):

$$
\nabla \times \mathbf{B}-\frac{1}{c^{2}} \frac{\partial \mathbf{E}}{\partial t}=\mu_{0} \mathbf{j}
$$

and for $a=0$ and $b$ varying from 1 to 3 (inclusive) for the inhomogeneous Maxwell equation

$$
\boldsymbol{\nabla} \cdot \mathbf{E}=\rho / \epsilon_{0} .
$$

The homogeneous Maxwell equations are presented by (7). Using (A27) and for $a=1, c=2$, and $b=3$ results in

$$
\boldsymbol{\nabla} \cdot \mathbf{B}=\mathbf{0}
$$

and for the values $a=0$ and $b$ and $c$ varying from 1 to 3 (inclusive), (7) results in

$$
\nabla \times \mathbf{E}=-\partial \mathbf{B} / \partial t
$$


In the absence of gravity the energy-momentum tensor (8) reduces for nonrelativistic units to

$$
T_{a b}=\frac{1}{\mu_{0}}\left[F_{a c} F_{c b}+\frac{1}{4} \delta_{a b} F_{c d} F^{c d}\right]
$$

Substitution of (A27) in (A33) gives the result that $T_{\infty}$ equals the electromagnetic energy density $w$ :

$$
T_{00}=\frac{1}{2}\left[\epsilon_{0} E^{2}+\frac{B^{2}}{\mu_{0}}\right]=w
$$

and for the terms $\left(T_{01}, T_{02}, T_{03}\right)$ :

$$
\left(T_{01}, T_{02}, T_{03}\right)=\frac{-i}{c}\left(S_{x}, S_{y}, S_{z}\right)=\frac{-i}{c} \mathrm{~S}
$$

where $\mathbf{S}$ is the Poynting vector, $\mathbf{S}=\mathbf{E} \times \mathbf{B} / \mu_{0}$. In the absence of external confining forces, the row $i c T_{a b}$ (for $a=0$ ) in the tensor (A33), transforms like a (pseudo-) 4-vector and is introduced as the Poynting 4-vector in (36), which modulus is Lorentz-invariant under inertial movements relative to the observer.

Substitution of the Lorentz-invariant quantities interval $d s=$ $\left(-x^{a} x^{a}\right)^{1 / 2}$, rest mass $m_{0}$, speed of light $c$, scalar product $A^{a} x^{a}$, and electric charge $q$ in the action integral $S$ results in

$$
S=\int_{1}^{t_{1}}\left[-m_{0} c d s+q(\mathbf{A} \cdot d \mathbf{r}-\varphi d t)\right]
$$

Separating the time interval $d t$ in (A36) results in

$S=\int_{l_{1}}^{t_{2}}\left[-m_{0} c\left[\frac{1-v^{2}}{c^{2}}\right]^{1 / 2}+q(\mathbf{A} \cdot \mathbf{v}-\varphi)\right] d t=\int_{t_{1}^{2}}^{t_{2}} L d t$,

where $L$ is the Lagrangian. The action integral $S$ is zero over a small time interval $t_{1}-t_{2}$ when $L$ satisfies the Euler-Lagrange equation

$$
\frac{d}{d t}\left(\frac{\partial L}{\partial \mathbf{v}}\right)-\frac{\partial L}{\partial \mathbf{r}}=0
$$

Equation (A38) is split into two parts:

$$
\frac{\partial L}{\partial \nabla}=\frac{m_{0} \nabla}{\left(1-v^{2} / c^{2}\right)^{1 / 2}}+q \mathrm{~A}=p+q \mathrm{~A}
$$

and

$$
\frac{\partial L}{\partial \mathbf{r}}=\nabla L=q[\nabla(\mathbf{A} \cdot \mathbf{\nabla})-\nabla \varphi]
$$

Substituting (A39) and (A40) into (A38) leads to the Lorentzinvariant equation

$$
\frac{d \mathbf{p}}{d t}=-q \frac{\partial \mathbf{A}}{\partial t}-q \nabla \varphi+q[\mathbf{\nabla} \times(\nabla \times \mathbf{A})]
$$

Substituting (1) and (2) in (A41) gives the Lorentz-invariant equation for the mechanical force $F$ acting on a charged mass in an electromagnetic field:

$$
\mathbf{F}=\frac{d p}{d t}=q(\mathbf{E}+\mathbf{v} \times \mathbf{B})
$$

The current 4-vector $\boldsymbol{f}^{2}$ is defined as

$$
J^{a}=q v^{a}=q\left(i c, v_{x}, v_{y}, v_{z}\right)
$$

The current density 4-vector is defined in (5) and is presented in a similar way as (A43):

$$
j^{a}=(i c \rho, \mathbf{j})=\rho v^{a}=\rho\left(i c, v_{x}, v_{y}, v_{z}\right)
$$

The force density 3-vector $f$ is now defined, according to (A42), as

$$
\mathbf{f}=\rho(\mathbf{E}+\nabla \times \mathbf{B})
$$

Then the force density 4-vector can be presented as

$$
f^{a}=-F^{a b} j^{b}
$$

which follows from substitution of (A27) and (A44) in (A46) and gives a similar result as Eq. (A45). Substituting (A28) in (A46) yields

$$
f^{a}=\frac{1}{\mu_{0}} F^{a b} \partial_{c} F_{b c}=\partial_{b} T^{a b}
$$

From (A47) it follows that in three dimensions the mechanical force $F$ acting on an arbitrary electromagnetic confinement equals 


$$
\mathbf{F}=\int \mathbf{f} d V=\int_{\text {ol }} \nabla \cdot \stackrel{\overline{\mathbf{T}}}{\mathbf{T}} d V=\int_{\text {suthace }} n \cdot \stackrel{\overline{\mathbf{T}}}{\mathrm{T}} d S
$$

For that reason the energy-momentum tensor is often presented by

$$
T^{a b}=\left|\begin{array}{cc}
w & \frac{-i}{c} \mathrm{~S} \\
\frac{-i}{c} \mathrm{~S} & =
\end{array}\right|
$$

where the tension subtensor $\bar{t}$ describes the force density acting on the electromagnetic confinement. The momentum 4-vector in (33) is presented by

$$
p^{a}=\frac{i}{c} \int_{\text {vol }} T^{a} d V
$$

which equation equals (39) in the article. In this example a simplified energy-momentum tensor is chosen that describes the confinement of a monochromatic electromagnetic wave, presented in (A8), which propagates along the $x$-axis between perfect reflecting mirrors, which counterbalance the radiation pressure due to the confinement. This balance is, for example, realized by putting an opposite electric charge on both perfect reflecting mirrors. When the energy density $w_{s}=1 / 2 E_{x}^{2}$ of this static electric field equals the energy density $w_{D}$ of the confined electromagnetic radiation, averaged over the period time, the system is in balance, and under that restriction the Lorentz transformation of the energy-momentum tensor is allowed. The energy-momentum tensor equals

$$
T^{a b}=\left|\begin{array}{cccc}
w_{S}+w_{D} & \frac{-i}{c} S_{x} & 0 & 0 \\
\frac{-i}{c} S_{x} & w_{s}-w_{D} & 0 & \epsilon_{0} E_{x} E_{z} \\
0 & 0 & T_{22} & 0 \\
0 & \epsilon_{0} E_{x} E_{z} & 0 & T_{33}
\end{array}\right|
$$

where $w_{s}$ is the static energy density due to the electric charge on both confining mirrors and equals

$$
w_{s}=1 / 2 \epsilon_{0} E_{x}^{2},
$$

and $w_{D}$ is the dynamic energy density of the confined electromagnetic radiation, propagating along the $x$-axis:

$$
w_{D}=\frac{1}{2} \epsilon_{0} E_{z}^{2}+\frac{1}{2 \mu_{0}} B_{Y}^{2} .
$$

The term $T_{22}$ equals

$$
T_{22}=\frac{1}{\mu_{0}} B_{y}^{2}-w_{S}-w_{D},
$$

and the term $T_{33}$ equals

$$
T_{33}=\epsilon_{0} E_{z}^{2}-w_{s}-w_{D}
$$

From (A51) it follows that the trace $T^{\text {aa }}$ of the energymomentum tensor is zero. The Lorentz transformation of (A52) is described by

$$
T^{\alpha \prime^{\prime}}=L_{a}^{c} T^{a b} L_{b}^{d}
$$

in which the tensor $L_{a}^{c}$ equals

$$
L_{a}^{c}=\left|\begin{array}{cccc}
\gamma & \frac{i \gamma v}{c} & 0 & 0 \\
\frac{-i \gamma v}{c} & \gamma & 0 & 0 \\
0 & 0 & 1 & 0 \\
0 & 0 & 0 & 1
\end{array}\right|,
$$

and the tensor $L_{b}^{d}$ equals

$$
L_{b}^{d}=\left|\begin{array}{cccc}
\gamma & \frac{-i \gamma \nu}{c} & 0 & 0 \\
\frac{i \gamma \nu}{c} & \gamma & 0 & 0 \\
0 & 0 & 1 & 0 \\
0 & 0 & 0 & 1
\end{array}\right| .
$$

Substitution of (A51), (A57), and (A58) in (A56) results in the term $T_{00}^{\prime}$ of the transformed energy-momentum tensor

$$
T_{00}^{\prime}=w_{D}^{\prime}+w_{S}^{\prime}=\gamma^{2}\left[\left(1+\frac{v^{2}}{c^{2}}\right] w_{D}+\frac{w_{s}}{\gamma^{2}}-\frac{2 v S_{x}}{c^{2}}\right]
$$

and for the transformed term $T_{10}^{\prime}$

$$
T_{10}^{\prime}=-\frac{i}{c} S_{x}^{\prime}=-\frac{i \gamma^{2}}{c}\left[\left[1+\frac{v^{2}}{c^{2}}\right] S_{x}-2 v w_{D}\right]
$$

The quantity $S_{X}$ describes the dynamic part of the Poynting vector and is indicated in the article as $S_{D x}$. Then (A60) can be 
written as

$$
S_{D X}^{\prime}+S_{S X}^{\prime}=\gamma^{2}\left[\left(1+\frac{v^{2}}{c^{2}}\right] S_{D X}+\frac{S_{S x}}{\gamma^{2}}-2 v w_{D}\right] \text {. }
$$

The transformations (A59) and (A61) are presented in Eq. (41). The 0 component of Eq. (A47) equals

$$
f^{0}=-F^{a b} j^{b}=\partial_{b} T^{0 b}
$$

Using (A46) to determine $f^{0}$ and substituting (A33) in (A62) results in the Poynting theorem, better known as the continuity equation

$$
\mathbf{E} \cdot \mathbf{J}=-\frac{\partial w}{\partial t}-\nabla \cdot \mathbf{S}
$$

In the absence of gravity and in a perfect vacuum the left-hand side of (A62) equals zero. In the article it was demonstrated [Eq. (56)] that the Schrödinger equation is a special notation for

$$
\partial_{b} T^{\infty b}=0
$$

at nonrelativistic velocities, so that the energy and the momentum are observed as separated quantities in which circumstance the Schrödinger equation controls the energy domain.

Received 14 January 1994.

\begin{abstract}
Résumé
Dans le domaine d'un modele électromagnétique de la matière, le présent exposé suppose que les ondes de probabilité mécanique quantique ne constituent que les effets relativistes observés d'ondes électromagnétiques (auto-) confinées. Un couplage relativiste dans le domaine énergétique de l'équation de continuité aboutit à l'équation d'onde de Schrödinger, tandis qu'un couplage étendu intégrant le domaine du couple (observé comme un "spin") aboutit à l'équation de Dirac. On démontre au chapitre 3 que l'équation (relativiste) de Dirac a vraisemblablement trouvé son origine dans les équations de Maxwell. Dans le cadre de ce modèle où cette matiere électromagnétique, la "lumière", ou plus généralement des "radiations électromagnétiques", est considérée comme le matériau constitutif de la matière, ce qui va effectivement à l'encontre de l'idée que la lumiere est constituée de particules elémentaires (photons). Ce concept postulé d'inversion est étendu aux particules de masse résiduelle finie. Les conditions de stabilité requises pour les auto-confinements électromagnétiques (EONs) sont obtenues à l'aide de forces gravitationnelles ou électromagnétiques ou une combinaison de celles-ci pour le contróle du confinement.
\end{abstract}

\section{References}

1. J. Wheeler, Phys. Rev. 97, 511 (1955).

2. W.G.V. Rosser, Classical Electromagnetism via Relativity (Butterworths, London, 1968), p. 134.

3. M. Molski, Phys. Essays 6, 143 (1993).

4. R.C. Jennison, J. Phys. A: Math. Gen. 13, 2247 (1980).

5. Ibid. 16, 3635 (1983).

6. R.C. Jennison and A.J. Drinkwater, ibid. 10, 167 (1977).

7. R.C. Jennison, ibid. 11, 1525 (1978).

8. H. Jehle, Phys. Rev. D 3, 306 (1971).

9. Ibid. 11, 2147 (1975).

10. R.C. Jennison, M.A.C. Jennison, and T.M.C. Jennison, J. Phys. A: Math. Gen. 19, 2249 (1986).

11. M. Molski, ibid. 26, 1765 (1993).

12. A.O. Barut, Phys. Lett. A 169, 120 (1992).

13. M. Molski, J. Phys. A: Math. Gen. 24, 5063 (1991).

14. C. Elbaz, Phys. Lett. A 127, 308 (1988).

15. Ibid., 123, 205 (1987).

16. W. Pauli, Wissenschaftlicher Briefwechsel mit Einstein, Bohr, Heisenberg (Springer Verlag, NY, 1985).

17. H. Bethe and R. Jackiw, Intermediate Quantum Mechanics
(Benjamin Cummings, Menlo Park, 1986).

18. K. Gottfried and V. Weisskopf, Concepts of Particle Physics (Oxford University Press, Oxford, 1986).

19. H. Jehle, Phys. Rev. D 6, 441 (1972).

20. M. Kaku, Introduction to Superstrings (Springer Verlag, NY, 1988).

21. P. Freund, Introduction to Supersymmetry (Cambridge University Press, NY, 1986).

22. R. Feynman and S. Weinberg, Elementary Particles and the Laws of Physics (Cambridge University Press, Cambridge, 1986).

23. H.B.G. Casimir, Physica 19, 846 (1953).

24. A.D. Yaghjian, in Proceedings of the URSI International Symposium on Electromagnetic Theory, Int. Union Radio Sci. 14 (17), 322 (1989).

25. A.D. Yaghjian, A Direct Approach to the Derivation of Electric Dyadic Green's Functions, NBS TN 1000, Washington, 1977.

26. E. Power and J. Wheeler, Rev. Mod. Phys. 29, 480 (1957).

27. W. Petrich, J. Opt. Soc. Am. B 11 (8), 1332 (1994).

28. Y.B. Ovchinnikov, Opt. Spectrosc. 76 (2), 188 (1994). 
29. M. Zaluzny, Physica B 128, 171 (1985).

30. R.C. Jennison, J. Phys. A: Math. Gen. 15, 405 (1982).

31. R. Spreeuw and J. Woerdman, in Proceedings of the International Symposium on the Analogies in Optics and Micro-Electronics, special volume of Physica B 175, ISSN 0921-4526 (Eindhoven, North-Holland, 1991).

32. R. Chiao, P. Kwiat, and A. Steinberg, in Ref. 31.

33. A. Einstein, Relativity: the Special and the General Theory (Methuen, London, 1920).

\section{J.W. Vegt}

HOZN-PTH Department of Micro System Technology

Campus University TUE Het Eeuwsel 2

Postbus 826

5600-AV Eindhoven, The Netherlands 